\title{
A TOA-AOA-Based NLOS Error Mitigation Method for Location Estimation
}

\author{
Hong Tang, ${ }^{1}$ Yongwan Park, ${ }^{1}$ and Tianshuang Qiu ${ }^{2}$ \\ ${ }^{1}$ Mobile Communication Laboratory, Yeungnam University, kyongsan, kyongbuk 712-749, South Korea \\ ${ }^{2}$ School of Electronic and Information Engineering, Dalian University of Technology, Liaoning 116024, China
}

Correspondence should be addressed to Yongwan Park, ywpark@yu.ac.kr

Received 28 February 2007; Revised 21 July 2007; Accepted 31 October 2007

Recommended by Sinan Gezici

\begin{abstract}
This paper proposes a geometric method to locate a mobile station (MS) in a mobile cellular network when both the range and angle measurements are corrupted by non-line-of-sight (NLOS) errors. The MS location is restricted to an enclosed region by geometric constraints from the temporal-spatial characteristics of the radio propagation channel. A closed-form equation of the MS position, time of arrival (TOA), angle of arrival (AOA), and angle spread is provided. The solution space of the equation is very large because the angle spreads are random variables in nature. A constrained objective function is constructed to further limit the MS position. A Lagrange multiplier-based solution and a numerical solution are proposed to resolve the MS position. The estimation quality of the estimator in term of "biased" or "unbiased" is discussed. The scale factors, which may be used to evaluate NLOS propagation level, can be estimated by the proposed method. AOA seen at base stations may be corrected to some degree. The performance comparisons among the proposed method and other hybrid location methods are investigated on different NLOS error models and with two scenarios of cell layout. It is found that the proposed method can deal with NLOS error effectively, and it is attractive for location estimation in cellular networks.
\end{abstract}

Copyright (c) 2008 Hong Tang et al. This is an open access article distributed under the Creative Commons Attribution License, which permits unrestricted use, distribution, and reproduction in any medium, provided the original work is properly cited.

\section{INTRODUCTION}

Wireless location for a mobile station (MS) in a cellular network has gained tremendous attention in the last decade due to support from the Federal Communication Commission (FCC) and wide range of potential applications using location-based information. Accurate positioning is already considered as one of the essential features of third generation $(3 \mathrm{G})$ wireless systems in winning a wide acceptance. Most location techniques depend on the measurements of time of arrival (TOA), received signal strength (RSS), time difference of arrival (TDOA), and/or angle of arrival (AOA) [1-5]. For TOA location methods, the TOA measurement provides a circle centered at the base station (BS) on which the MS must lie. The MS location estimate is determined by the intersection of circles; at least three BSs are involved in the location process to resolve ambiguities arising from multiple crossing of the positioning lines. The RSS-based location has the same trilateration concept if the propagation path losses are transformed into distances. For TDOA location methods, the distance differences of the MS to at least three BSs are mea- sured. Each TDOA measurement provides a hyperbolic locus on which the MS must lie and the position estimate is determined by the intersection of two or more hyperbolas. For AOA location methods, the angle of arrival of the MS to the BS is measured by multielement antenna array or multibeamforming antenna. A line from the MS to the BS can be drawn according to each AOA measurement and the position of the MS is calculated from the intersection of at least two lines. High accuracy can be derived from these methods with the assumption of line-of-sight (LOS) propagation. However, location errors will inevitably increase greatly as the assumption is violated by NLOS.

NLOS can cause different range error in different propagation environments, from dozen of meters to thousands of meters [6]. In open area there are almost no obstacles. The signal travels in LOS. However, in mountains, urban environments, or bad urban environments, the signal may transmit in reflection, diffraction; and it takes longer for the signal to arrive at the receiver. As a result the range error caused by NLOS is always a positive number. In [7], LOS was reconstructed using the statistics of the measurement 
data. Mathematical programming techniques were adopted in $[5,8-10]$ to evaluate the NLOS propagation effect. No prior knowledge was required in [8-10], but the variance of measurement noise was required in [5]. A method to determine and identify the number of line-of-sight BSs based on a residual test was proposed in [11]. In [12], a geometric location method was proposed. It can suppress NLOS error to some degree. Scattering-model-based methods were proposed to classify propagation environments via moment matching, expectation maximization, and Bayesian estimation in [13]. The bias of time measures in NLOS environment was tracked with Kalman filter in [14], and the evaluation of the approach was carried out in real scenarios.

From the basic principle of the time-based location methods [8-12], we know that they are valid only when at least three BSs can support the location process. However, this requirement may not always be met at all times because of the hearability limitation of an MS, that is, the ability of a mobile to listen to a BS. The field trials in [6] conducted in a GSM network showed that over $92 \%$ of the time in urban environments and $71 \%$ of the time in rural environments, three or more BSs can be received by the MS. However, for two or more BSs, the corresponding percentages are $98 \%$ and $95 \%$, for each environment. This means that about $6 \%$ of the time in urban environments and $24 \%$ of the time in rural environments only two BSs can support an MS. Or in some cases, more BSs can support an MS, but only two BSs have high reliability for location purpose. Or somewhere, the BSs are sparse and only two BSs may be available. Thus the time-based location methods will suffer from ambiguity.

Hybrid location methods by combining time measurement and angle measurement can reduce the number of receiving BSs and improve the coverage of location-based service in cellular network simultaneously. The reference $[4,15-$ 19] proposed hybrid location methods which can be applied when only two BSs are involved. The AOA data fusion in [4] combined TOAs and AOAs into a group of linear equations. In [15], by taking advantage of two TOAs seen at the two BSs and AOA seen at home BS, the author proposed geometry-constrained location estimation (GLE) method to estimate the MS position. In [16], hybrid lines of position (HLOP) method were proposed, which combined the linear lines of position (LOP) generated by differencing pairs of squared range estimates and the linear LOP given by the AOA. In [17], two equations were built. One equation was built from TDOA and the other was built from AOA. Because closed form solution of the two equations is quit complex, a new coordinate system was constructed to simplify the two equations. Reference [18] focused on AOA-based location method which selected the two most reliable AOAs among the whole set of AOA measurements. The TOA-based historical data was used in [19] to resolve location ambiguity and Kalman filter was adopted to track trajectory. We note that these methods [15-17] only take advantage of the AOA seen at home BS. Other hybrid location methods can be found in [20-22]. And all of them [4, 15-22] seldom consider the temporal-spatial characteristics of the radio propagation channel.
This paper proposes an NLOS mitigation method motivated by the temporal-spatial characteristics of the radio channel. The geometric explanation of the method is presented in Section 2, including the temporal-spatial channel models, TOA, and AOA measurements. The mathematic model is given in Section 3, including the constraints derived from TOAs, AOAs, and angle spreads, and the construction of objective function. The solution and analysis to the model are presented in Section 4, including the Lagrangebased solution and estimation quality in terms of "biased" or "unbiased." The case of three BSs is discussed in Section 5. And the numerical solution is presented in Section 6. Computer simulation results are presented in Section 7 to show the performance, and remarks and conclusions are provided in Section 8.

\section{GEOMETRIC EXPLANATION TO THE PROPOSED METHOD}

\subsection{The temporal-spatial channel models of the propagation channel}

The temporal-spatial channel model can provide both delay spread and angle spread statistics of the channel. The angle spread is dependent on the wireless propagation environment. In a macrocell, the antenna height at the MS is low. The scatters surrounding the MS are about the same height or are higher than the MS. This results in the MS-received signal arriving from all directions after bouncing from surrounding scatters. AOA seen at the MS can be modeled as a random variable uniformly distributed over $[0,2 \pi]$. On the other hand, the antenna height at the BS is much higher than the surrounding scatters. The BS may not receive multipath reflections from locations near the BS. The received signal at the BS mainly comes from the scattering process in the vicinity of the mobile. AOA seen at the BS is restricted to a small angular region. And it is no longer uniformly distributed over $[0,2 \pi]$. A circular mode was proposed in [23] to describe the joint TOA/AOA probability density function (pdf) as seen in Figure 1, where the scatters are assumed to be uniformly distributed in a circle and an MS is the center of the circle. This is the so-called circular disk of scatters model (CDSM). For example, when the distance from MS to $\mathrm{BS}$ is 1000 meters and the scattering radius is 200 meters, the marginal TOA pdf and the marginal AOA pdf are shown in Figures 2(a) and 2(b), respectively. It can be found that the absolute angle spread is within 11 degrees and the excess delay is within 1.4 microseconds. In a microcell, both the antenna heights at BSs and MSs are low. The scatters are near the BS and the MS. An elliptical model is used to model this propagation environment. The scatters are assumed to be uniform distributed in an ellipse where the BS and the MS are the two foci. AOA seen at the BS has a larger angle spread. But it is also found that the joint AOA/TOA components seen at the BS are concentrated near line-of-sight [23]. The measurement campaigns reported in [24] are consistent with the above CDSM. It is suggested that AOA seen at the BS in a macrocell is like a Gaussian distribution with typical standard deviation of angle spreads approximately 6 degrees, and 


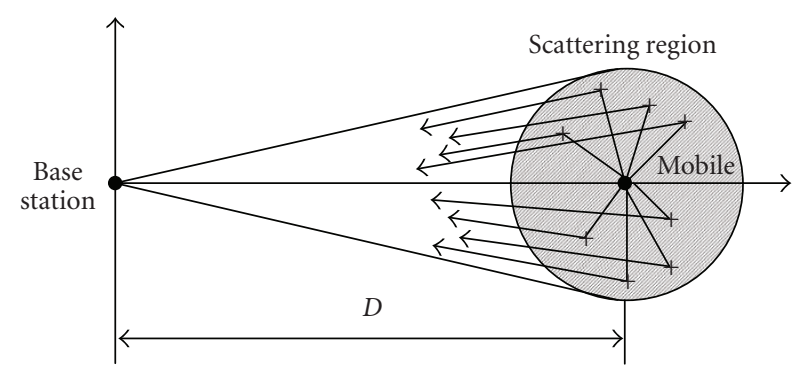

Figure 1: Circular scatter geometry for a macrocell.

the delay spread can be described by an exponential distribution. Further discussion about angle spread can be found in [25] and the references therein. The temporal-spatial characteristics of the propagation channel derived in [24] tend to be consistent with the results in $[23,25]$.

The other models are used to study delay spread is the ring of scatters model (ROSM) $[13,26]$ and the distancedependent model (DDM) [10,27]. The ROSM is also a classical model to describe macrocellular environments where the scatters are uniformly distributed on a ring which is centered about the MS. In the DDM, the delay spread is taken to be proportional to the LOS distance. The paper [27] cited measurement results from Motorola and Ericsson that report a relationship between the mean excess delay $\tau_{m}$ and the rootmean-square (rms) delay spread $\tau_{\text {rms }}$ of the form $\tau_{m}=k \tau_{\text {rms }}$, where $k$ is proportionality constant. The observation and results from [28] also suggested that NLOS errors may increase with distance.

\subsection{The measurements on TOA and $A O A$ in cellular network}

The scheme to perform range measurement may be different in different systems. In the TDMA system, the time delay between the MS and the serving BS must be known to avoid overlapping time slots. This is called timing advance (TA). For example, TA is available in GSM and TD-SCDMA. TA can be used to approximate the distance between the serving BS and the MS. In the CDMA system, time delay can be estimated by coarse timing acquisition with a sliding correlator or fine timing acquisition with a delay loop lock (DLL). The later is better suited for a location system, as illustrated in [29]. Round trip delay (RTD) is a general method to determine the distance between the transmitter and receiver, which needs a time stamp when the signal is transmitted and a time stamp when the signal is received. The range is approximated by the time difference of the two time stamps. So, it is technically easy to approximate the distance between the MS and the serving BS. In this paper, it is firstly assumed that only two BSs can support the MS, that is, BS1 and BS2 as shown in Figure 3. BS1 is the serving BS. In order to get the distance to the other BS, the so-called "force handover" could be a good choice [6]. When the locating is to be done, the network will force the MS to make a handover attempt from the serving BS to the other BS. Usually the other BS is the closest neighbor BS. The neighbor BS will measure the TA argu- ment in the TDMA system or the time delay in the CDMA system (or other techniques are taken to perform range measurement) and then reject the handover request. The range measurement $r_{i}$ between an MS to the BSi is expressed as

$$
r_{i}=c \cdot\left(t_{\mathrm{d}, i}+t_{\mathrm{e}, i}\right), \quad i=1,2,
$$

where $c$ is the speed of light, $t_{\mathrm{d}, i}$ is the LOS path delay to the BSi, and $t_{\mathrm{e}, i}$ is the excess delay caused by NLOS. The two TOA measurements provide two circles. Because $t_{\mathrm{e}, i}$ is always a positive number, the MS position must be in an area overlapped by the two circles, as shown in Figure 3 .

With the introduction of smart antenna array into wireless communication networks, the AOA of each MS can be estimated. Usually the subspace-based AOA estimation algorithms have good accuracy and resolution, such as the MUSIC [30] and ESPRIT algorithms. Multibeam antennas reported in [31] were also used to estimate AOA of an MS. For example, in TD-SCDMA technical specification [32], the precision of $\mathrm{AOA}$ for location purposes was required to be 15 degrees. In the NLOS environment the transmitted signal could only reach the receiver through reflected, diffracted, or scattered paths. Thus the AOA observed at the BS is not the exact LOS path AOA. The AOA measurement mainly consists two parts, which can be expressed as

$$
\theta_{i}=\theta_{\mathrm{d}, i}+\phi_{i}, \quad i=1,2,
$$

where $\theta_{\mathrm{d}, i}$ is the LOS path AOA and $\phi_{i}$ is the angle spread caused by NLOS propagation, which can be accurately described by a Gaussian random variable in a macrocell or outdoor environment. The standard deviation of the Gaussian distribution can be predicted by theoretical model or calculated from experimental data. Therefore, it is possible to know the angular bounds from the statistics of the AOA distribution of a given NLOS environment. That is, the LOS path AOA must be in an interval with a certain high confidence level. It is assumed that $\theta_{\min , i}$ and $\theta_{\max , i}$ are corresponding upper and lower bounds. Then the following inequality holds (or holds with a sufficiently high confidence level):

$$
\theta_{\min , i} \leq \theta_{\mathrm{d}, i} \leq \theta_{\max , i}, \quad i=1,2 .
$$

For example, if the angle spread seen at BSi is modeled as a Gaussian distribution $N\left(0, \sigma^{2}\right), \theta_{\mathrm{d}, i}$ must be in the interval $\left[\theta_{i}-2 \sigma, \theta_{i}+2 \sigma\right]$ with confidence level 95.4\%, where $\sigma$ is the standard deviation. Therefore, the MS position is further constrained to a small enclosed region overlapped by the two circles and the angular bounds, as illustrated in Figure 3, that is, the estimated MS position must satisfy the following restrictions:

$$
\begin{gathered}
\hat{r}_{\mathrm{d}, i} \leq r_{i}, \quad i=1,2, \\
\theta_{\min , i} \leq \hat{\theta}_{\mathrm{d}, i} \leq \theta_{\text {max }, i}, \quad i=1,2,
\end{gathered}
$$

where $\hat{r}_{\mathrm{d}, i}$ and $\hat{\theta}_{\mathrm{d}, i}$ are the estimates of LOS range and LOS $\mathrm{AOA}$ between the $\mathrm{BS} i$ and the MS, respectively.

TOA and AOA in a microcell can be described by an elliptical model [23], where the AOA measurement tends to 


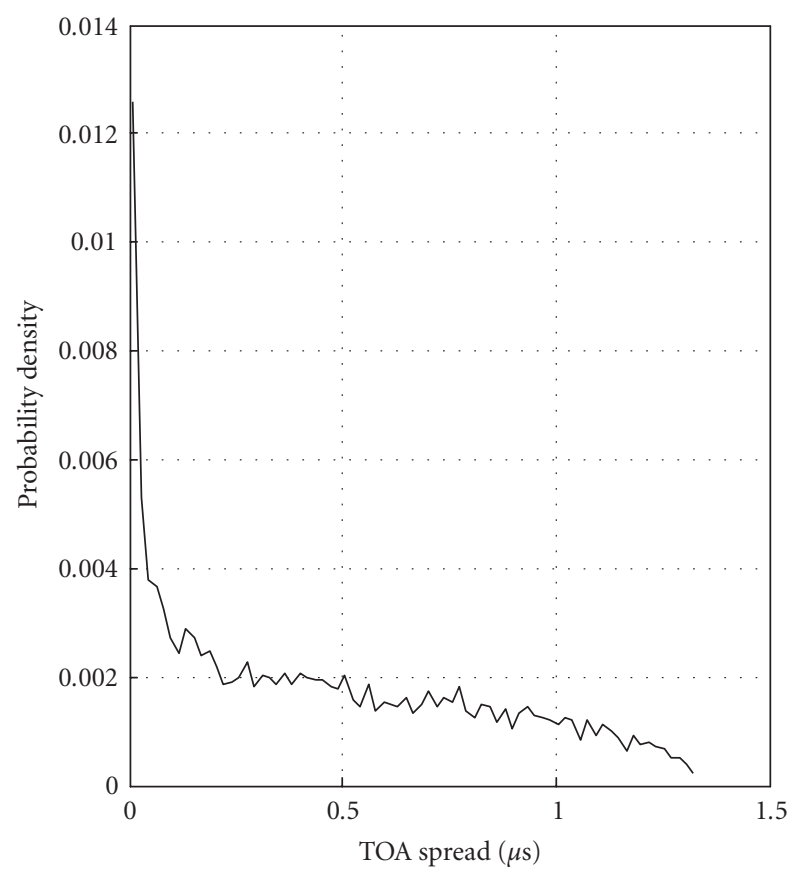

(a)

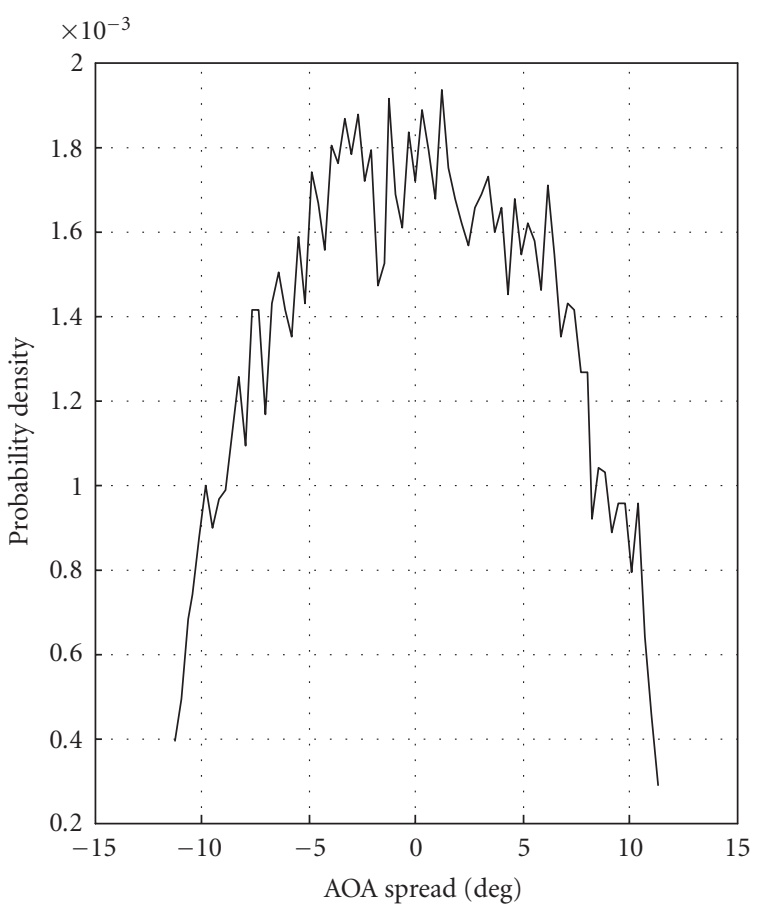

(b)

FIGURE 2: The temporal-spatial characteristics of the propagation channel in a macrocell where the distance from the MS to the BS is 1000 meters and the scatter radius is 200 meters. (a) The marginal TOA pdf and (b) the marginal AOA pdf.

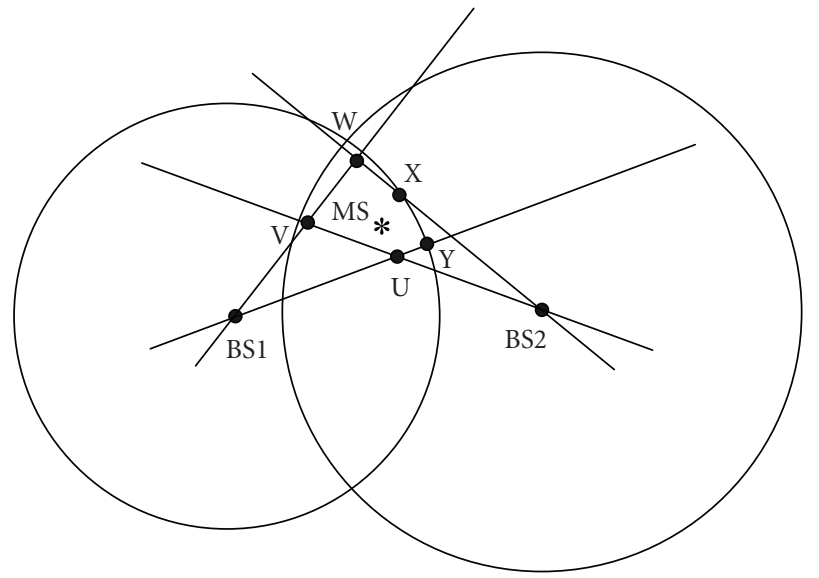

FIGURE 3: Geometry constraints from the temporal-spatial channel show that the MS lies in the overlapped region.

have large spread over $[0,2 \pi]$. Fortunately, the cell coverage is small in a microcell environment and more than two BSs may be received. The time-based location methods will not suffer from ambiguity.

\section{MATHEMATICAL MODEL TO THE MS POSITION ESTIMATION}

\subsection{Constraints from TOA measurements}

The location process is considered in a two-dimensional (2D) space and two BSs are involved. Let $\left(x_{0}, y_{0}\right)$ be the MS position to be determined and let $\left(x_{i}, y_{i}\right)$ be the coordinate of $\mathrm{BS} i$, where $i=1,2 . r_{i}$ is the range measurement. In the NLOS propagation environment, $r_{i}$ is always larger than the LOS range. The following inequality must hold:

$$
\sqrt{\left(x_{0}-x_{i}\right)^{2}+\left(y_{0}-y_{i}\right)^{2}} \leq r_{i}, \quad i=1,2 .
$$

In order to change the inequality (5) into equality, let the variable $\alpha_{i}$ be the scale factor of $r_{i}$. $\alpha_{i}$ must be constrained to

$$
\eta_{i} \leq \alpha_{i} \leq 1,
$$

where $\eta_{1}=\left(R-r_{2}\right) / r_{1}$ and $\eta_{2}=\left(R-r_{1}\right) / r_{2}$. $R$ is the distance between the two BSs. We get

$$
\sqrt{\left(x_{0}-x_{i}\right)^{2}+\left(y_{0}-y_{i}\right)^{2}}=\alpha_{i} r_{i}, \quad i=1,2 .
$$

Define the 2D function $d_{i}(x, y)$ which expresses the distance between the position $(x, y)$ and the BSi:

$$
d_{i}(x, y)=\sqrt{\left(x-x_{i}\right)^{2}+\left(y-y_{i}\right)^{2}}, \quad i=1,2 .
$$

If $\left(x_{s}, y_{s}\right)$ is an approximation position of the true MS position, the function $d_{i}(x, y)$ can be expanded in Taylor's series:

$$
\begin{gathered}
d_{i}(x, y) \approx d_{i}\left(x_{s}, y_{s}\right)+\left.\frac{\partial d_{i}(x, y)}{\partial x}\right|_{\substack{x=x_{s} \\
y=y_{s}}}\left(x-x_{s}\right) \\
+\left.\frac{\partial d_{i}(x, y)}{\partial y}\right|_{\substack{x=x_{s} \\
y=y_{s}}}\left(y-y_{s}\right) .
\end{gathered}
$$


In (9), only the terms of zero-order and first-order are kept. Let $\boldsymbol{\omega}_{0}=\left[x_{0}, y_{0}\right]^{\mathrm{T}}$ and $\mathbf{C}\left(\boldsymbol{\omega}_{0}\right)=\left[d_{1}\left(\boldsymbol{\omega}_{0}\right), d_{2}\left(\boldsymbol{\omega}_{0}\right)\right]^{\mathrm{T}}$ be the distance vectors. According to $(9), \mathbf{C}\left(\boldsymbol{\omega}_{0}\right)$ can be written as

$$
\mathbf{C}\left(\omega_{0}\right) \approx \mathbf{C}\left(\boldsymbol{\omega}_{s}\right)+\mathbf{H}\left(\boldsymbol{\omega}_{s}\right)\left(\boldsymbol{\omega}_{0}-\boldsymbol{\omega}_{s}\right)
$$

$\mathbf{H}\left(\boldsymbol{\omega}_{s}\right)$ is the gradient matrix

$$
\mathbf{H}\left(\boldsymbol{\omega}_{s}\right)=\left[\begin{array}{ll}
\frac{x_{s}-x_{1}}{d_{1}\left(\boldsymbol{\omega}_{s}\right)} & \frac{y_{s}-y_{1}}{d_{1}\left(\boldsymbol{\omega}_{s}\right)} \\
\frac{x_{s}-x_{2}}{d_{2}\left(\boldsymbol{\omega}_{s}\right)} & \frac{y_{s}-y_{2}}{d_{2}\left(\boldsymbol{\omega}_{s}\right)}
\end{array}\right]
$$

From (7) and (10), an equality that describes a linear range model incorporating NLOS errors can be expressed as

$$
\mathbf{H}\left(\boldsymbol{\omega}_{s}\right) \cdot \boldsymbol{\omega}_{0}-\mathbf{L}=\mathbf{H}\left(\boldsymbol{\omega}_{s}\right) \cdot \boldsymbol{\omega}_{s}-\mathbf{C}\left(\boldsymbol{\omega}_{s}\right),
$$

where $\mathbf{L}=\left[\alpha_{1} r_{1}, \alpha_{2} r_{2}\right]^{\mathrm{T}}$ is the corrected distance vector. Equation (12) can be rearranged as

$$
\left[\begin{array}{cccc}
\frac{x_{s}-x_{1}}{d_{1}\left(\boldsymbol{\omega}_{s}\right)} & \frac{y_{s}-y_{1}}{d_{1}\left(\boldsymbol{\omega}_{s}\right)} & -r_{1} & 0 \\
\frac{x_{s}-x_{2}}{d_{2}\left(\boldsymbol{\omega}_{s}\right)} & \frac{y_{s}-y_{2}}{d_{2}\left(\boldsymbol{\omega}_{s}\right)} & 0 & -r_{2}
\end{array}\right]\left[\begin{array}{l}
x_{0} \\
y_{0} \\
\alpha_{1} \\
\alpha_{2}
\end{array}\right]=\mathbf{H}\left(\boldsymbol{\omega}_{s}\right) \cdot \boldsymbol{\omega}_{s}-\mathbf{C}\left(\boldsymbol{\omega}_{s}\right) .
$$

\subsection{Constraints from AOA measurements}

It is assumed that the two BSs and the MS are in a coordinate system as shown in Figure 4 . AOA seen at BS1 is $\theta_{1}$ and the corresponding angle spread is $\phi_{1}$. AOA seen at BS2 is $\theta_{2}$ and the corresponding angle spread is $\phi_{2}$. From the geometry relationships shown in Figure 4, it can be found that the equation $\mathrm{CB}=\mathrm{CA}-\mathrm{BA}$ holds where $\mathrm{CB}=r_{\mathrm{d}, 1} \sin \left(\phi_{1}\right)$, $\mathrm{CA}=x_{0} \sin \left(\theta_{1}\right)$, and $\mathrm{BA}=y_{0} \cos \left(\theta_{1}\right)$. We obtain the following equation:

$$
x_{0} \sin \left(\theta_{1}\right)-y_{0} \cos \left(\theta_{1}\right)=r_{\mathrm{d}, 1} \sin \left(\phi_{1}\right),
$$

where $r_{\mathrm{d}, 1}$ is the LOS distance between the MS and BS1. It can be rewritten as $r_{\mathrm{d}, 1}=\alpha_{1} r_{1}$. Therefore, (14) can be modified to

$$
x_{0} \sin \left(\theta_{1}\right)-y_{0} \cos \left(\theta_{1}\right)-\alpha_{1} r_{1} \sin \left(\phi_{1}\right)=0 .
$$

Similarly, the equation ED $=\mathrm{EA}-\mathrm{DA}$ holds, where EA = $\left(x_{2}-x_{0}\right) \sin \left(\pi-\theta_{2}\right), \mathrm{DA}=y_{0} \cos \left(\pi-\theta_{2}\right)$, and $\mathrm{ED}=$ $\alpha_{2} r_{2} \sin \left(\phi_{2}\right)$. The following equation holds:

$$
x_{0} \sin \left(\theta_{2}\right)-y_{0} \cos \left(\theta_{2}\right)-\alpha_{2} r_{2} \sin \left(\phi_{2}\right)=x_{2} \sin \left(\theta_{2}\right) \text {. }
$$

Combining (15) and (16) gives

$$
\begin{gathered}
{\left[\begin{array}{cccc}
\sin \left(\theta_{1}\right) & -\cos \left(\theta_{1}\right) & -r_{1} \sin \left(\phi_{1}\right) & 0 \\
\sin \left(\theta_{2}\right) & -\cos \left(\theta_{2}\right) & 0 & -r_{2} \sin \left(\phi_{2}\right)
\end{array}\right]\left[\begin{array}{c}
x_{0} \\
y_{0} \\
\alpha_{1} \\
\alpha_{2}
\end{array}\right]} \\
\quad=\left[\begin{array}{c}
0 \\
x_{2} \sin \left(\theta_{2}\right)
\end{array}\right] .
\end{gathered}
$$

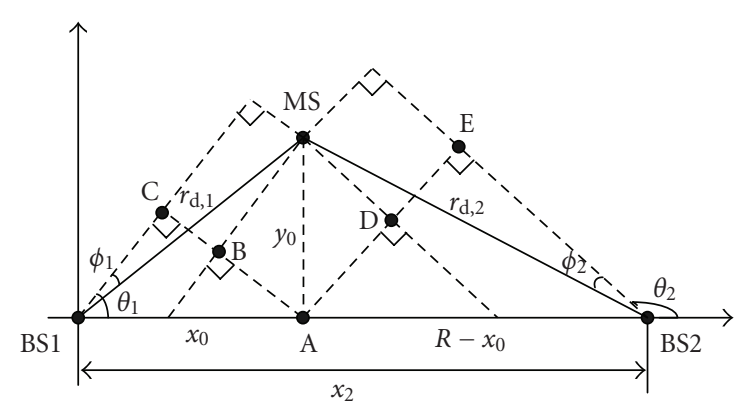

Figure 4: Constraints from AOA measurements.

\subsection{MS position estimation}

Combining (13) and (17) into a single matrix-vector form gives

$$
\mathbf{A}_{4 \times 4} \boldsymbol{\omega}_{4 \times 1}^{\prime}=\mathbf{b}_{4 \times 1}
$$

where

$$
\begin{aligned}
\mathbf{A}_{4 \times 4} & =\left[\begin{array}{llcc}
\frac{x_{s}-x_{1}}{d_{1}\left(\boldsymbol{\omega}_{s}\right)} & \frac{y_{s}-y_{1}}{d_{1}\left(\boldsymbol{\omega}_{s}\right)} & -r_{1} & 0 \\
\frac{x_{s}-x_{2}}{d_{2}\left(\boldsymbol{\omega}_{s}\right)} & \frac{y_{s}-y_{2}}{d_{2}\left(\boldsymbol{\omega}_{s}\right)} & 0 & -r_{2} \\
\sin \left(\theta_{1}\right) & -\cos \left(\theta_{1}\right) & -r_{1} \sin \left(\phi_{1}\right) & 0 \\
\sin \left(\theta_{2}\right) & -\cos \left(\theta_{2}\right) & 0 & -r_{2} \sin \left(\phi_{2}\right)
\end{array}\right] \\
\boldsymbol{\omega}_{4 \times 1}^{\prime} & =\left[\begin{array}{llll}
x_{0} & y_{0} & \alpha_{1} & \alpha_{2}
\end{array}\right]^{\mathrm{T}} \\
\mathbf{b}_{4 \times 1} & =\left[\begin{array}{llll}
B_{1} & B_{2} & 0 & x_{2} \sin \left(\theta_{2}\right)
\end{array}\right]^{\mathrm{T}}
\end{aligned}
$$

where $B_{1}$ denotes $\left(x_{s}-x_{1} / d_{1}\left(\boldsymbol{\omega}_{s}\right)\right) x_{s}+\left(y_{s}-y_{1} / d_{1}\left(\boldsymbol{\omega}_{s}\right)\right) y_{s}-$ $d_{1}\left(\boldsymbol{\omega}_{s}\right), B_{2}$ denotes $\left(x_{s}-x_{2} / d_{2}\left(\boldsymbol{\omega}_{s}\right)\right) x_{s}+\left(y_{s}-y_{2} / d_{2}\left(\boldsymbol{\omega}_{s}\right)\right) y_{s}-$ $d_{2}\left(\omega_{s}\right)$. Equation (18) is a closed-form equation of the MS position, TOAs, AOAs, and angle spreads. Now perform inverse operation on $\mathbf{A}$. We can estimate the MS position and scale factors as

$$
\widehat{\boldsymbol{\omega}}^{\prime}=\mathbf{A}^{-1} \mathbf{b}
$$

The estimated position by (20) is very accurate if the angle spreads are sufficiently small or the angle spreads are prior known. However, $\phi_{1}$ and $\phi_{2}$ are independent random variables in nature as well as $\alpha_{1}$ and $\alpha_{2}$ because the two BSs are spatial separately by large distance, and the propagation environments experienced by the emitted signals from the MS are totally different. So, it is impossible to know the angle spreads accurately. For example, $\phi_{1}$ and $\phi_{2}$ have probability density function on CDSM, as shown in the right plot of Figure 2.

Therefore, the solution space of (20) becomes large due to the unknown angle spreads. In order to determine the MS position, an objective function must be taken to further limit the MS position. Here, the objective function is taken to minimize the sum of the square of the distance from the MS position to the all endpoints of the enclosed region 
overlapped by the two circles and angular bounds, as shown in Figure 3;

$$
J\left(\boldsymbol{\omega}_{0}\right)=\sum_{j=1}^{K}\left\|\boldsymbol{\omega}_{0}-\boldsymbol{\lambda}_{j}\right\|^{2}
$$

is subject to

$$
\mathbf{A} \boldsymbol{\omega}^{\prime}=\mathbf{b}, \quad \eta_{i} \leq \alpha_{i} \leq 1,
$$

where $\boldsymbol{\lambda}_{j}=\left[x_{j, x}, y_{j, y}\right]^{\mathrm{T}}$ is the coordinate of the end points (points $\mathrm{U}, \mathrm{V}, \mathrm{W}, \mathrm{X}$, and $\mathrm{Y}$ in Figure 3 ). $K$ is the total number of all endpoints and $\|\cdot\|$ is the 2 -norm operator. From the geometry explanation in Section 2, we know that the end points $\mathrm{U}, \mathrm{V}, \mathrm{W}, \mathrm{X}$, and $\mathrm{Y}$ are, indeed, the nearest points to the MS. So, it is reasonable to restrict the MS position by using the objective function. It must be noted that the total number of all end points of the enclosed region is not fixed. It is a variable depending on TOA measurements and angular bounds.

\section{SOLUTION AND ANALYSIS}

\subsection{The solution by using the technique of Lagrange multiplier}

The objective function (21) can be modified to

$$
J^{\prime}\left(\boldsymbol{\omega}^{\prime}\right)=\boldsymbol{\omega}^{, \mathrm{T}} \mathbf{M} \boldsymbol{\omega}^{\prime}+\mathbf{b}_{m}^{\mathrm{T}} \boldsymbol{\omega}^{\prime}+\sum_{j=1}^{K}\left(x_{j, x}^{2}+y_{j, y}^{2}\right),
$$

where

$$
\begin{aligned}
\mathbf{M} & =\left[\begin{array}{cccc}
K & 0 & 0 & 0 \\
0 & K & 0 & 0 \\
0 & 0 & 0 & 0 \\
0 & 0 & 0 & 0
\end{array}\right], \\
\mathbf{b}_{m} & =\left[\begin{array}{llll}
-2 \sum_{j=1}^{K} x_{j, x} & -2 \sum_{j=1}^{K} y_{j, x} & 0 & 0
\end{array}\right]^{\mathrm{T}} .
\end{aligned}
$$

The constrained problem can be solved by using the technique of Lagrange multipliers and the Lagrangian to be minimized is

$$
\begin{aligned}
L\left(\boldsymbol{\omega}^{\prime}, \rho\right) & =J^{\prime}\left(\boldsymbol{\omega}^{\prime}\right)+\rho\left(\mathbf{A} \boldsymbol{\omega}^{\prime}-\mathbf{b}\right)^{\mathrm{T}}\left(\mathbf{A} \boldsymbol{\omega}^{\prime}-\mathbf{b}\right) \\
& =\boldsymbol{\omega}^{\prime \mathrm{T}}\left(\mathbf{M}+\rho \mathbf{A}^{\mathrm{T}} \mathbf{A}\right) \boldsymbol{\omega}^{\prime}+\left(\mathbf{b}_{m}^{\mathrm{T}}-2 \rho \mathbf{b}^{\mathrm{T}} \mathbf{A}\right) \boldsymbol{\omega}^{\prime},
\end{aligned}
$$

where $\rho$ is the Lagrange multiplier to be determined. The derivative of an estimate of $L\left(\boldsymbol{\omega}^{\prime}\right)$ with respect to $\boldsymbol{\omega}^{\prime}$ is

$$
\frac{\partial L\left(\boldsymbol{\omega}^{\prime}\right)}{\partial \boldsymbol{\omega}^{\prime}}=2\left(\mathbf{M}+\rho \mathbf{A}^{\mathrm{T}} \mathbf{A}\right) \boldsymbol{\omega}^{\prime}+\left(\mathbf{b}_{m}^{\mathrm{T}}-2 \rho \mathbf{b}^{\mathrm{T}} \mathbf{A}\right)^{\mathrm{T}} .
$$

Let the derivative equal zero. We obtain

$$
\omega^{\prime}=\left(2\left(\mathbf{M}+\rho \mathbf{A}^{\mathrm{T}} \mathbf{A}\right)\right)^{-1}\left(\mathbf{b}_{m}-2 \rho \mathbf{A}^{\mathrm{T}} \mathbf{b}\right) .
$$

At the same time, $\boldsymbol{\omega}^{\prime}$ must meet the constraint $\mathbf{A} \boldsymbol{\omega}^{\prime}=\mathbf{b}$, that is,

$$
\left(\mathbf{A} \boldsymbol{\omega}^{\prime}-\mathbf{b}\right)^{\mathrm{T}}\left(\mathbf{A} \boldsymbol{\omega}^{\prime}-\mathbf{b}\right)=0
$$

Substituting (27) into (28) yields

$$
\begin{aligned}
f\left(\boldsymbol{\omega}^{\prime}, \rho\right)= & \left(\mathbf{A}\left(\left(2\left(\mathbf{M}+\rho \mathbf{A}^{\mathrm{T}} \mathbf{A}\right)\right)^{-1}\left(\mathbf{b}_{m}-2 \rho \mathbf{A}^{\mathrm{T}} \mathbf{b}\right)\right)-\mathbf{b}\right)^{\mathrm{T}} \\
& \cdot\left(\mathbf{A}\left(\left(2\left(\mathbf{M}+\rho \mathbf{A}^{\mathrm{T}} \mathbf{A}\right)\right)^{-1}\left(\mathbf{b}_{m}-2 \rho \mathbf{A}^{\mathrm{T}} \mathbf{b}\right)\right)-\mathbf{b}\right) .
\end{aligned}
$$

Therefore, $\boldsymbol{\omega}^{\prime}$ and $\rho$ must be the root of (29). We use an iterative method to solve $\omega^{\prime}$ and $\rho$ as follows.

(i) Guess an initial position $\left[x_{0}(0), y_{0}(0)\right]$ and calculate $\alpha_{1}(0)$ and $\alpha_{2}(0)$. Let $\boldsymbol{\omega}^{\prime}(0)=\left[\begin{array}{llll}x_{0}(0) & x_{0}(0) & \alpha_{1}(0) & \alpha_{2}(0)\end{array}\right]^{\mathrm{T}}$ and $k=0$.

(ii) Combine $\boldsymbol{\omega}^{\prime}(k)$ and (27), we get $\boldsymbol{\omega}_{\rho}^{\prime}(k)$, where $\phi_{i}$ in $\mathbf{A}$ is $\phi_{i}=\theta_{i}-\tan ^{-1}\left[\left(y_{i}-y_{0}(k)\right) /\left(x_{i}-x_{0}(k)\right)\right]$.

(iii) Substitute $\boldsymbol{\omega}_{\rho}^{\prime}(k)$ into (29), we get $f\left(\boldsymbol{\omega}^{\prime}(k), \rho\right)$. Find a $\rho(k)$ which makes $f\left(\boldsymbol{\omega}^{\prime}(k), \rho(k)\right)<T$, where $T$ is a threshold.

(iv) Substitute $\rho(k)$ into (27), we get $\boldsymbol{\omega}^{\prime}(k+1)$.

(v) Repeat steps (ii) and (iv) until $\omega^{\prime}(k)$ converges.

$\left[x_{0}(0), y_{0}(0)\right]$ can be randomly selected in the enclosed region formed by $\mathrm{U}, \mathrm{V}, \mathrm{W}, \mathrm{X}, \mathrm{Y}$, and $\mathrm{Z}$, as shown in Figure 3. It is impossible to find a $\rho(k)$ that can exactly make $f\left(\boldsymbol{\omega}^{\prime}(k), \rho(k)\right)=0$ because the position in each iteration contains error. So, we set up a threshold $T$. The simulation shows that $\rho(k)$ is a relative large number at the beginning. With the iteration going on, $\rho(k)$ becomes smaller and smaller.

\subsection{Estimation quality}

The proposed method is investigated in term of "biased" or "unbiased" in this subsection. A is divided into subblocks $\mathbf{A}=\left[\begin{array}{ll}\mathbf{A}_{11} & \mathbf{A}_{12} \\ \mathbf{A}_{21} & \mathbf{A}_{22}\end{array}\right] . \mathbf{A}_{11}, \mathbf{A}_{12}, \mathbf{A}_{21}$, and $\mathbf{A}_{22}$ are the corresponding subblocks of A:

$$
\begin{gathered}
\mathbf{A}_{11}=\left[\begin{array}{cc}
\frac{x_{s}-x_{1}}{d_{1}\left(\boldsymbol{\omega}_{s}\right)} & \frac{y_{s}-y_{1}}{d_{1}\left(\boldsymbol{\omega}_{s}\right)} \\
\frac{x_{s}-x_{2}}{d_{2}\left(\boldsymbol{\omega}_{s}\right)} & \frac{y_{s}-y_{2}}{d_{2}\left(\boldsymbol{\omega}_{s}\right)}
\end{array}\right], \quad \mathbf{A}_{12}=\left[\begin{array}{cc}
-r_{1} & 0 \\
0 & -r_{2}
\end{array}\right], \\
\mathbf{A}_{21}=\left[\begin{array}{cc}
\sin \left(\theta_{1}\right) & -\cos \left(\theta_{1}\right) \\
\sin \left(\theta_{2}\right) & -\cos \left(\theta_{2}\right)
\end{array}\right] \\
\mathbf{A}_{22}=\left[\begin{array}{cc}
-r_{1} \sin \left(\phi_{1}\right) & 0 \\
0 & -r_{2} \sin \left(\phi_{2}\right)
\end{array}\right]
\end{gathered}
$$

The matrix inverse $\mathbf{A}^{-1}$ can be inverted blockwise by using the following analytic inversion formula:

$$
\left[\begin{array}{ll}
\mathbf{A}_{11} & \mathbf{A}_{12} \\
\mathbf{A}_{21} & \mathbf{A}_{22}
\end{array}\right]^{-1}=\left[\begin{array}{ll}
Q_{1} & Q_{2} \\
Q_{3} & Q_{4}
\end{array}\right]
$$

where $Q_{1}$ denotes $\mathbf{A}_{11}^{-1}+\mathbf{A}_{11}^{-1} \mathbf{A}_{12}\left(\mathbf{A}_{22}-\mathbf{A}_{21} \mathbf{A}_{11}^{-1} \mathbf{A}_{12}\right)^{-1} \mathbf{A}_{21} \mathbf{A}_{11}^{-1}$, $Q_{2}$ denotes $-\mathbf{A}_{11}^{-1} \mathbf{A}_{12}\left(\mathbf{A}_{22}-\mathbf{A}_{21} \mathbf{A}_{11}^{-1} \mathbf{A}_{12}\right)^{-1}, \quad Q_{3}$ denotes - $\left(\mathbf{A}_{22}-\mathbf{A}_{21} \mathbf{A}_{11}^{-1} \mathbf{A}_{12}\right)^{-1} \mathbf{A}_{21} \mathbf{A}_{11}^{-1}$, and $Q_{4}$ denotes $\left(\mathbf{A}_{22}-\right.$ $\left.\mathbf{A}_{21} \mathbf{A}_{11}^{-1} \mathbf{A}_{12}\right)^{-1}$. If $\phi_{1}$ and $\phi_{2}$ are sufficiently small and have zero mean, that is, the expectation of $\mathbf{A}_{22}$ is $\mathrm{E}\left(\mathbf{A}_{22}\right)=\mathbf{0}_{2 \times 2}$, as 


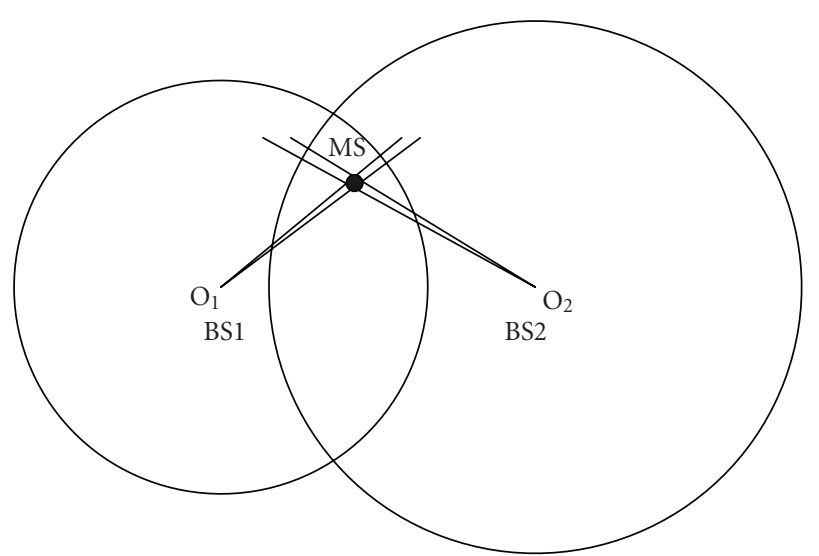

FIGURE 5: MS location estimation when the angle spreads are sufficiently small.

shown in Figure 5. Note that $\left(\mathbf{A}_{21} \mathbf{A}_{11}^{-1} \mathbf{A}_{12}\right)^{-1}=\mathbf{A}_{12}^{-1} \mathbf{A}_{11} \mathbf{A}_{21}^{-1}$. According to (31), the expectation of $\mathbf{A}^{-1}$ can be arranged as

$$
\mathrm{E}\left(\mathbf{A}^{-1}\right)=\left[\begin{array}{cc}
\mathbf{0}_{2 \times 2} & \mathrm{E}\left(\mathbf{A}_{21}^{-1}\right) \\
\mathbf{A}_{12}^{-1} & -\mathbf{A}_{12}^{-1} \mathbf{A}_{11} \mathrm{E}\left(\mathbf{A}_{21}^{-1}\right)
\end{array}\right] .
$$

From (20), we know $\mathrm{E}\left(\widehat{\boldsymbol{\omega}}^{\prime}\right)=\mathrm{E}\left(\mathbf{A}^{-1} \mathbf{b}\right)$. Substitute (32) into this equation and note that $\mathrm{E}\left(\theta_{1}\right)=\theta_{\mathrm{d}, 1}$ and $\mathrm{E}\left(\theta_{2}\right)=\theta_{\mathrm{d}, 2}$, the expectation of $\left(x_{0}, y_{0}\right)$ can be expressed as follows:

$$
\begin{aligned}
\mathrm{E}\left(x_{0}\right) & =\frac{1}{\left(-\sin \theta_{\mathrm{d}, 1} \cos \theta_{\mathrm{d}, 2}+\cos \theta_{\mathrm{d}, 1} \sin \theta_{\mathrm{d}, 2}\right)} x_{2} \cos \theta_{\mathrm{d}, 1} \sin \theta_{\mathrm{d}, 2} \\
& =\frac{1}{-\sin \left(\theta_{\mathrm{d}, 1}-\theta_{\mathrm{d}, 2}\right)} x_{2} \cos \theta_{\mathrm{d}, 1} \sin \theta_{\mathrm{d}, 2}, \\
\mathrm{E}\left(y_{0}\right) & =\frac{1}{\left(-\sin \theta_{\mathrm{d}, 1} \cos \theta_{\mathrm{d}, 2}+\cos \theta_{\mathrm{d}, 1} \sin \theta_{\mathrm{d}, 2}\right)} x_{2} \sin \theta_{\mathrm{d}, 1} \sin \theta_{\mathrm{d}, 2} \\
& =\frac{1}{-\sin \left(\theta_{\mathrm{d}, 1}-\theta_{\mathrm{d}, 2}\right)} x_{2} \sin \theta_{\mathrm{d}, 1} \sin \theta_{\mathrm{d}, 2} .
\end{aligned}
$$

We note that (33) is also the exact intersection of the lines drawn by $\theta_{\mathrm{d}, 1}$ and $\theta_{\mathrm{d}, 2}$, that is,

$$
\mathrm{E}\left(y_{0}\right)=y_{0}, \quad \mathrm{E}\left(x_{0}\right)=x_{0} .
$$

This means that the estimator is unbiased as long as the angle spreads are sufficiently small with zero mean and $\sin \left(\theta_{\mathrm{d}, 1}-\right.$ $\left.\theta_{\mathrm{d}, 2}\right) \neq 0$.

When $\sin \left(\theta_{\mathrm{d}, 1}-\theta_{\mathrm{d}, 2}\right)=0$, the MS position must be on $\overline{\mathrm{O}_{1} \mathrm{O}_{2}}$ as shown in Figure 6. According to the objective function, the estimate of the MS position $\hat{x}_{0}$ is the center of $\overline{\mathrm{GH}}$ :

$$
\hat{x}_{0}=\frac{\overline{O_{1} O_{2}}-r_{2}+r_{1}}{2}=\frac{R-r_{\mathrm{d}, 2}-r_{\mathrm{e}, 2}+r_{\mathrm{d}, 1}+r_{\mathrm{e}, 1}}{2},
$$

where $r_{\mathrm{e}, i}$ is the NLOS error seen at BSi. The true MS position is $x_{0}=r_{\mathrm{d}, 1}$. So, the location error is

$$
\hat{x}_{0}-x_{0}=\frac{R-r_{\mathrm{d}, 2}-r_{\mathrm{e}, 2}+r_{\mathrm{d}, 1}+r_{\mathrm{e}, 1}}{2}-r_{\mathrm{d}, 1}=\frac{r_{\mathrm{e}, 1}-r_{\mathrm{e}, 2}}{2} .
$$

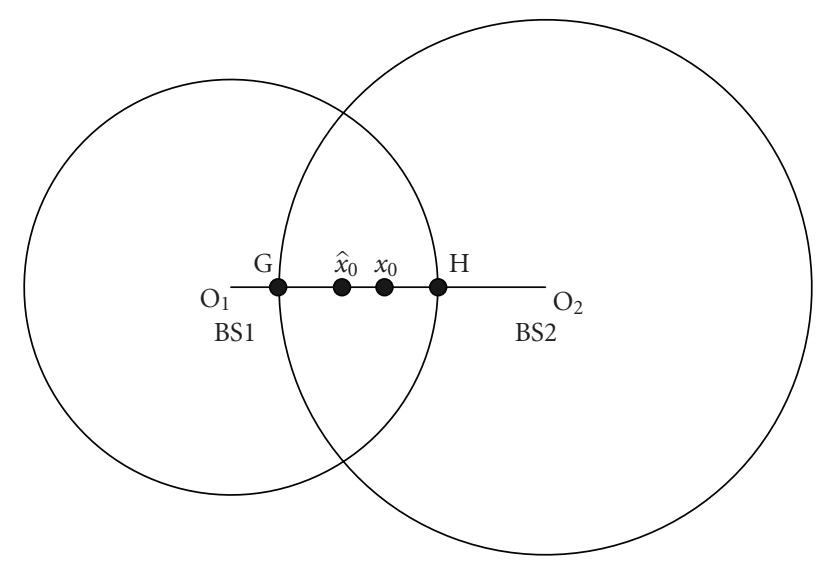

FIgURE 6: Location estimation when $\sin \left(\theta_{\mathrm{d}, 1}-\theta_{\mathrm{d}, 2}\right)=0$.

Note that $R=r_{\mathrm{d}, 1}+r_{\mathrm{d}, 2}$, then the expectation of the location error is

$$
\begin{aligned}
\mathrm{E} & \left(\hat{x}_{0}-x_{0}\right) \\
& =\frac{1}{2}\left(\mathrm{E}\left(r_{\mathrm{e}, 1}\right)-\mathrm{E}\left(r_{\mathrm{e}, 2}\right)\right) \\
& =\frac{1}{2}\left(\int_{-\infty}^{\infty} r_{\mathrm{e}, 1} p_{1}\left(r_{\mathrm{e}, 1}\right) d\left(r_{\mathrm{e}, 1}\right)-\int_{-\infty}^{\infty} r_{\mathrm{e}, 2} p_{2}\left(r_{\mathrm{e}, 2}\right) d\left(r_{\mathrm{e}, 2}\right)\right),
\end{aligned}
$$

where $p_{1}(\cdot)$ and $p_{2}(\cdot)$ are NLOS error probability density function observed at BS1 and BS2, respectively.

Therefore, we conclude that the proposed estimator is

(i) unbiased if $\sin \left(\theta_{\mathrm{d}, 1}-\theta_{\mathrm{d}, 2}\right) \neq 0$,

(ii) unbiased if $\sin \left(\theta_{\mathrm{d}, 1}-\theta_{\mathrm{d}, 2}\right)=0$ and $p_{1}(\cdot)=p_{2}(\cdot)$,

(iii) biased if $\sin \left(\theta_{\mathrm{d}, 1}-\theta_{\mathrm{d}, 2}\right)=0$ and $p_{1}(\cdot) \neq p_{2}(\cdot)$, and the bias is $(1 / 2)\left(\mathrm{E}\left(r_{\mathrm{e}, 1}\right)-\mathrm{E}\left(r_{\mathrm{e}, 2}\right)\right)$,

while the angle spreads are sufficiently small and have zero mean.

\subsection{Selection of the approximate position}

In the solution procedure, the approximation position $\boldsymbol{\omega}_{s}$ must be predefined. A simplest choice is the position defined by TOA and AOA measurements seen at BS1 in a polar coordinate system where the origin is the position of BS1:

$$
\begin{aligned}
& x_{s}=r_{1} \cos \left(\theta_{1}\right), \\
& y_{s}=r_{1} \sin \left(\theta_{1}\right) .
\end{aligned}
$$

Besides this choice, there are some other choices, such as the intersection of lines drawn by $\theta_{1}$ and $\theta_{2}$, the AOA data fusion in [4], and so forth.

\section{THE CASE OF THREE BASE STATIONS}

The proposed location method can be extended to more than 2 BSs scenario. As an example, three BSs are involved in the location process, as seen in Figure 7. BS3 is the third BS. The 
position $\mathrm{O}\left(x_{0}, y_{0}\right)$ is the MS location. The range measurement and angle measurement at BS3 are $r_{3}$ and $\theta_{3}$, respectively. From the geometry found in Figure 7 , we find that $\mathrm{GF}=\mathrm{GO}_{3}-\mathrm{FO}_{3}$, where $\mathrm{FO}_{3}=r_{\mathrm{d}, 3} \cos \left(\theta_{3}+\phi_{3}-\pi / 2\right)=$ $\alpha_{3} r_{3} \cos \left(\theta_{3}+\phi_{3}-\pi / 2\right), \mathrm{GF}=y_{0}$, and $\mathrm{GO}_{3}=y_{3}$, that is, we get the following equation:

$$
y_{0}=y_{3}-\alpha_{3} r_{3} \cos \left(\theta_{3}+\phi_{3}-\frac{\pi}{2}\right)
$$

Similarly, $\mathrm{GH}=\mathrm{GO}_{1}-\mathrm{HO}_{1}$ where $\mathrm{GH}=r_{\mathrm{d}, 3} \sin \left(\theta_{3}+\phi_{3}-\right.$ $\pi / 2)=\alpha_{3} r_{3} \sin \left(\theta_{3}+\phi_{3}-\pi / 2\right), \mathrm{GO}_{1}=x_{3}$, and $\mathrm{HO}_{1}=x_{0}$, that is,

$$
x_{0}=x_{3}-\alpha_{3} r_{3} \sin \left(\theta_{3}+\phi_{3}-\frac{\pi}{2}\right) \text {. }
$$

Combining (39) and (40) yields

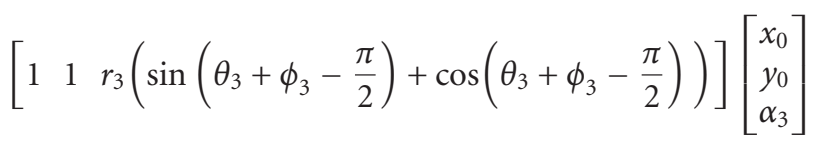

$$
\begin{aligned}
& =x_{3}+y_{3} \text {. }
\end{aligned}
$$

Combining (41) and the angular constraints from BS1 and BS2, that is, (17), yields

$$
\begin{gathered}
{\left[\begin{array}{ccccc}
\sin \left(\theta_{1}\right) & -\cos \left(\theta_{1}\right) & -r_{1} \sin \left(\phi_{1}\right) & 0 & 0 \\
\sin \left(\theta_{2}\right) & -\cos \left(\theta_{2}\right) & 0 & -r_{2} \sin \left(\phi_{2}\right) & 0 \\
1 & 1 & 0 & 0 & V
\end{array}\right]\left[\begin{array}{c}
x_{0} \\
y_{0} \\
\alpha_{1} \\
\alpha_{2} \\
\alpha_{3}
\end{array}\right]} \\
\quad=\left[\begin{array}{c}
0 \\
x_{2} \sin \left(\theta_{2}\right) \\
x_{3}+y_{3}
\end{array}\right],
\end{gathered}
$$

where $V$ denotes $r_{3}\left(\sin \left(\theta_{3}+\phi_{3}-\pi / 2\right)+\cos \left(\theta_{3}+\phi_{3}-\pi / 2\right)\right)$. For the case of three BSs, the constraints from TOA measurements (13) can be directly extended to

$$
\begin{gathered}
{\left[\begin{array}{lllll}
\frac{x_{s}-x_{1}}{d_{1}\left(\boldsymbol{\omega}_{s}\right)} & \frac{y_{s}-y_{1}}{d_{1}\left(\boldsymbol{\omega}_{s}\right)} & -r_{1} & 0 & 0 \\
\frac{x_{s}-x_{2}}{d_{2}\left(\boldsymbol{\omega}_{s}\right)} & \frac{y_{s}-y_{2}}{d_{2}\left(\boldsymbol{\omega}_{s}\right)} & 0 & -r_{2} & 0 \\
\frac{x_{s}-x_{3}}{d_{3}\left(\boldsymbol{\omega}_{s}\right)} & \frac{y_{s}-y_{3}}{d_{3}\left(\boldsymbol{\omega}_{s}\right)} & 0 & 0 & -r_{3}
\end{array}\right]\left[\begin{array}{l}
x_{0} \\
y_{0} \\
\alpha_{1} \\
\alpha_{2} \\
\alpha_{3}
\end{array}\right]} \\
=\left[\begin{array}{ll}
\frac{x_{s}-x_{1}}{d_{1}\left(\boldsymbol{\omega}_{s}\right)} & \frac{y_{s}-y_{1}}{d_{1}\left(\boldsymbol{\omega}_{s}\right)} \\
\frac{x_{s}-x_{2}}{d_{2}\left(\boldsymbol{\omega}_{s}\right)} & \frac{y_{s}-y_{2}}{d_{2}\left(\boldsymbol{\omega}_{s}\right)} \\
\frac{x_{s}-x_{3}}{d_{3}\left(\boldsymbol{\omega}_{s}\right)} & \frac{y_{s}-y_{3}}{d_{3}\left(\boldsymbol{\omega}_{s}\right)}
\end{array}\right]\left[\begin{array}{l}
x_{s} \\
y_{s}
\end{array}\right]-\left[\begin{array}{l}
d_{1}\left(\boldsymbol{\omega}_{s}\right) \\
d_{2}\left(\boldsymbol{\omega}_{s}\right) \\
d_{3}\left(\boldsymbol{\omega}_{s}\right)
\end{array}\right] .
\end{gathered}
$$

Combining (42) and (43) into a single matrix-vector form yields

$$
\mathbf{A}_{6 \times 5} \boldsymbol{\omega}_{5 \times 1}^{\prime}=\mathbf{b}_{6 \times 1}
$$

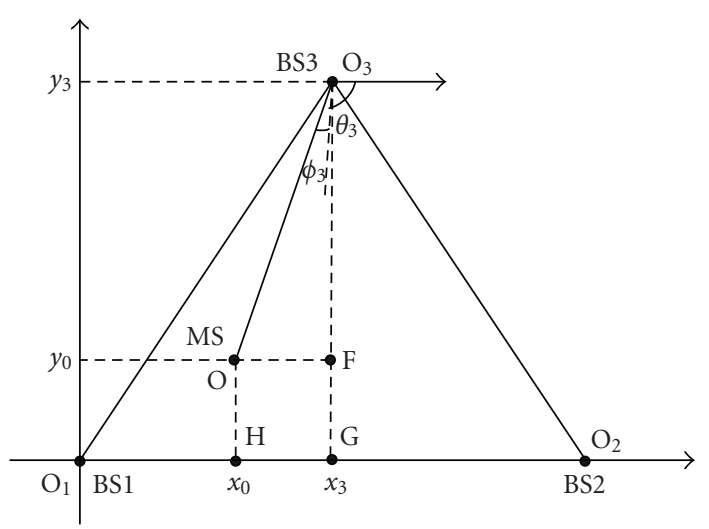

Figure 7: The constraint from AOA measurement with BS3.

where

$$
\begin{aligned}
& \mathbf{A}_{6 \times 5}=\left[\begin{array}{ccccc}
\sin \left(\theta_{1}\right) & -\cos \left(\theta_{1}\right) & -r_{1} \sin \left(\phi_{1}\right) & 0 & 0 \\
\sin \left(\theta_{2}\right) & -\cos \left(\theta_{2}\right) & 0 & -r_{2} \sin \left(\phi_{2}\right) & 0 \\
1 & 1 & 0 & 0 & N \\
\frac{x_{s}-x_{1}}{d_{1}\left(\boldsymbol{\omega}_{s}\right)} & \frac{y_{s}-y_{1}}{d_{1}\left(\boldsymbol{\omega}_{s}\right)} & -r_{1} & 0 & 0 \\
\frac{x_{s}-x_{2}}{d_{2}\left(\boldsymbol{\omega}_{s}\right)} & \frac{y_{s}-y_{2}}{d_{2}\left(\boldsymbol{\omega}_{s}\right)} & 0 & -r_{2} & 0 \\
\frac{x_{s}-x_{3}}{d_{3}\left(\boldsymbol{\omega}_{s}\right)} & \frac{y_{s}-y_{3}}{d_{3}\left(\boldsymbol{\omega}_{s}\right)} & 0 & 0 & -r_{3}
\end{array}\right], \\
& \boldsymbol{\omega}_{5 \times 1}^{\prime}=\left[\begin{array}{lllll}
x_{0} & y_{0} & \alpha_{1} & \alpha_{2} & \alpha_{3}
\end{array}\right]^{\mathrm{T}} \\
& \mathbf{b}_{6 \times 1}=\left[\begin{array}{llllll}
0 & x_{2} \sin \left(\theta_{2}\right) & x_{3}+y_{3} & D_{1} & D_{2} & D_{3}
\end{array}\right]^{\mathrm{T}} \text {, }
\end{aligned}
$$

where $N$ denotes $r_{3}\left(\sin \left(\theta_{3}+\phi_{3}-\pi / 2\right)+\cos \left(\theta_{3}+\phi_{3}-\pi / 2\right)\right)$; $D_{1}$ denotes $\left(\left(x_{s}-x_{1}\right) / d_{1}\left(\boldsymbol{\omega}_{s}\right)\right) x_{s}+\left(\left(y_{s}-y_{1}\right) / d_{1}\left(\boldsymbol{\omega}_{s}\right)\right) y_{s}-d_{1}\left(\boldsymbol{\omega}_{s}\right)$, $D_{2}$ denotes $\left(\left(x_{s}-x_{2}\right) / d_{2}\left(\boldsymbol{\omega}_{s}\right)\right) x_{s}+\left(\left(y_{s}-y_{2}\right) / d_{2}\left(\boldsymbol{\omega}_{s}\right)\right) y_{s}-d_{2}\left(\boldsymbol{\omega}_{s}\right)$, and $D_{3}$ denotes $\left(\left(x_{s}-x_{3}\right) / d_{3}\left(\boldsymbol{\omega}_{s}\right)\right) x_{s}+\left(\left(y_{s}-y_{3}\right) / d_{3}\left(\boldsymbol{\omega}_{s}\right)\right) y_{s}-$ $d_{3}\left(\omega_{s}\right)$.

Equation (44) is the closed-form equation of the MS position, TOAs, AOAs, angular spreads. The least-squares intermediate solution of (44) is

$$
\boldsymbol{\omega}^{\prime}=\left(\mathbf{A}^{\mathrm{T}} \mathbf{A}\right)^{-1} \mathbf{A}^{\mathrm{T}} \mathbf{b}
$$

As we discuss in former sections, the solution space of (46) is large due to the unknown angle spreads. The Lagrangedbased solution can be still applied here.

If either the AOA measurement or the TOA measurement is not available at BS3, the constraint (41) or the constraint from the TOA measurement will be absent. Equation (44) is reduced to

$$
\mathbf{A}_{5 \times 5} \boldsymbol{\omega}_{5 \times 1}^{\prime}=\mathbf{b}_{5 \times 1} .
$$

The location estimation process can be conducted in a similar way by the proposed method. 
If more than three BSs are involved in the location process, TOA and AOA can be available at each BS. Let the number of BSs be $n(n>3)$. Equation (44) can be extended to

$$
\mathbf{A}_{2 n \times(2+n)} \boldsymbol{\omega}_{(2+n) \times 1}^{\prime}=\mathbf{b}_{2 n \times 1},
$$

where $\boldsymbol{\omega}_{(2+n) \times 1}^{\prime}=\left[\begin{array}{lllll}x_{0} & y_{0} & \alpha_{1} & \cdots & \alpha_{n}\end{array}\right]^{\mathrm{T}} \cdot \mathbf{A}_{2 n \times(2+n)}$ and $\mathbf{b}_{2 n \times 1}$ are the corresponding matrixes that can be obtained in a similar manner. The least-square solution is

$$
\boldsymbol{\omega}_{(2+n) \times 1}^{\prime}=\left(\mathbf{A}_{2 n \times(2+n)}^{\mathrm{T}} \mathbf{A}_{2 n \times(2+n)}\right)^{-1} \mathbf{A}_{2 n \times(2+n)}^{\mathrm{T}} \mathbf{b}_{2 n \times 1} .
$$

In more than 2 BSs scenario, the time-based NLOS mitigation methods will not suffer from ambiguity. However, it is apparent that combing different types of the measurements can improve location performance. The computer simulations in Section 7 will show performance improvement with angle.

\section{NUMERICAL SOLUTION}

With the number of BSs increasing in the location process, the matrix $\mathbf{A}$ in (48) may be large, and (27) and $f\left(\boldsymbol{\omega}^{\prime}, \rho\right)$ become complex. It may be not easy to operate the matrix and perform the $\rho$ finding algorithm. A numerical solution is proposed to be an alternative to resolve the MS position, which is summarized in the following steps.

Step 1. The all-angle spreads from $\phi_{1}$ to $\phi_{n}$ are simulated by independent random variable sequences that satisfy the predefined distributions. The solution space of (49) can be denoted as a data set $\Pi_{1}=\left\{\widehat{\boldsymbol{\omega}}_{m}^{\prime}, 1 \leq m \leq M\right\}$. $M$ is the length of the sequence.

Step 2. If we constrain $\Pi_{1}$ by $\eta_{i} \leq \alpha_{i} \leq 1$, we get another data set $\Pi_{2}$.

Step 3. $\hat{\boldsymbol{\omega}}_{\mathrm{opt}}^{\prime}$ is the one in $\Pi_{2}$ that minimizes $J\left(\boldsymbol{\omega}_{0}\right)$, that is, $\hat{\boldsymbol{\omega}}_{\mathrm{opt}}^{\prime}=\min _{\boldsymbol{\omega}^{\prime} \in \Pi_{2}}\left\{J\left(\boldsymbol{\omega}^{\prime}\right)\right\}$.

In Step 1, each element in the solution space is a candidate of the MS position. In Steps 2 and 3, one of the candidates in $\Pi_{1}$, which can both meet the constraint $\eta_{i} \leq \alpha_{i} \leq 1$ and minimize $J\left(\boldsymbol{\omega}_{0}\right)$, is considered as the optimal position. The numerical solution is motivated by the constraints (18) or (48) and objective function.

From the numerical solution process, we know that most of the computation load happens in Step 1, and there are two factors that can affect the computation load. The first is the matrix inverse operation in (20) or (49). The second is the size of the solution space in Step 1, which is dependent on $M$. The computation load will linearly increase with $M$. As an example, the Gaussian elimination algorithm is used to calculate the matrix inverse, approximately $2 L^{3} / 3$ operations are needed, that is, the complexity of the matrix inverse is $O\left(L^{3}\right)$ where $L$ is the matrix size, $L=2 n$ and $n$ is the number of BSs involved in the location process. Simulations show that the position estimate by the numerical solution can be close to the position estimate by using the Lagrange-based solution, when $M$ is up to 100 .
For the Lagrange-based solution, both matrix multiplication and matrix inverse are needed in (27) in each iteration. The complexity of each iteration is also $\mathrm{O}\left(L^{3}\right)$ if matrix multiplication is carried out naively and finding $\rho$ is not considered. Simulations show that the total iteration number is usually 100. Therefore, we can conclude that the complexity of the numerical solution is comparable with that of the Lagrange-based solution.

\section{COMPUTER SIMULATIONS}

\subsection{TOA and AOA measurements}

When range measurements are performed in a system, other factors also can contribute range error, such as system delay, synchronization error, timing error, measurement noise, and so forth. System delay means that the system has to take time to process the received signal and prepare for the transmitting signal. For RTD measurement, system delay must be considered. For TOA measurement and time difference measurement, synchronization has great influence on range estimation. Cyclic synchronization is usually used to keep synchronization error in an acceptable level. For example, in the TD-SCDMA system, the technical specifications $[33,34]$ point out that synchronization resolution for location purposes should be limited within half chip, that is, about 100 meters. The timing error is caused by the uncorrected clock. With consideration of these factors, the TOA range measurement in the simulations is given as

$$
r_{i}=r_{\mathrm{d}, i}+v_{i}+\operatorname{nlos}_{i}
$$

where $v_{i}$ is the range error caused by these factors. $v_{i}$ is assumed to be a positive Gaussian random variable with mean 100 meters and standard variance 30 meters. nlos $_{i}$ is the excess distance due to NLOS propagation. The radius of the scatters of CDSM is assumed to be 200 meters, that is, nlos $_{i}$ are positive random variables having support over [0 400] meters. The NLOS range error models are shown in Figure 6. The other two models are reverse CDSM and uniform distribution. The reverse CDSM is used to study the performance in a high NLOS environment. If the probability density function (pdf) for CDSM is $f(\lambda)$, the pdf of the reverse CDMS is $f(400-\lambda)$.

The angle spread is modeled as Gaussian random variables. The standard deviation of the angle spread is 6 degrees, determined by the CDSM. The AOA measurement is as (2), and the angular bounds are as (3) which are selected with a confidence level of $95.4 \%$.

\subsection{Scenario 1: 2 BSs}

In this scenario, the performance of the proposed method will be examined with 2 BSs. The cell layout is shown in Figure 3. Let the coordinates of the two BSs be $(0,0)$ and $(R, 0)$, where $R=2000$ meters. The cell h radius is $R / 2$. The MS position is assumed to be uniformly distributed in right part of the serving cell. The performances of several methods are compared, including the proposed method, AOA data fusion in [4], the GLE in [15], the HLOP in [16], and the hybrid 


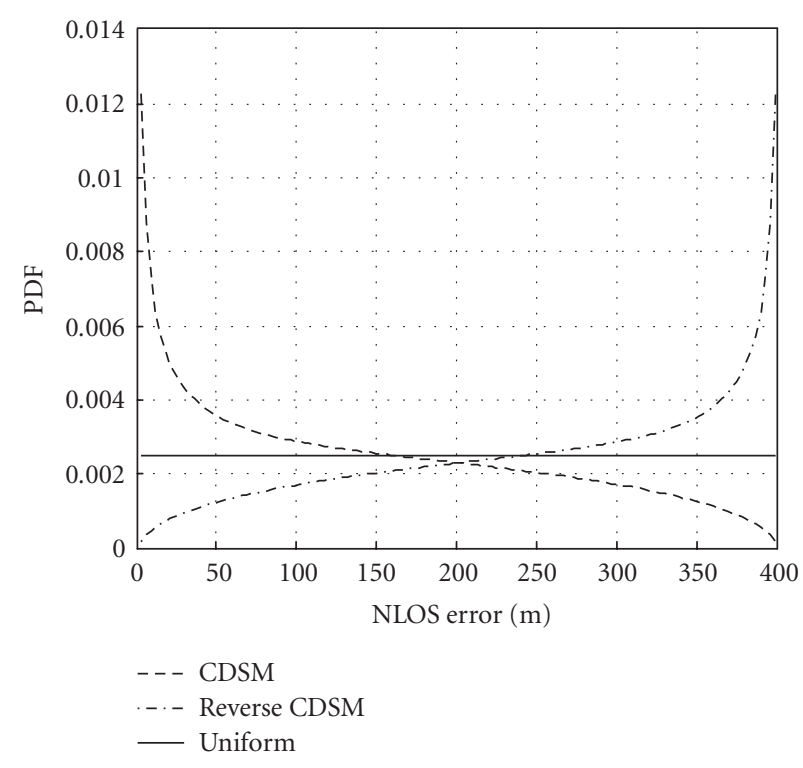

FIgURE 8: Probability density functions (pdfs) for the NLOS error models.

TDOA/AOA in [17]. The average location errors (ALEs) of these methods are shown in Figure 9. From the pdfs of the NLOS error models, we know that the reverse CDSM has a large probability with high NLOS error, that is, the reverse CDSM means a high NLOS environment, and vice versa for the CDSM. Uniform means medium NLOS error. As a result, it can be found that the ALEs of all the methods are smaller on the CDSM and the ALEs are larger on the reverse CDSM. The simulations show that the proposed method can effectively deal with NLOS error with two BSs.

The scale factor $\alpha_{i}$ also can be resolved by the proposed method. The scale factor reflects the approximation of range measurement to the LOS range. A high-scale factor means that the range measurement is close to the LOS range. A low scale factor means that the radio channel suffers from heavy NLOS propagation. The true scale factor of the range measurement seen at BSi is defined as $\alpha_{i}=c \cdot t_{\mathrm{d}, i} / r_{i}$. It is assumed that the MS position is uniformly distributed in the serving cell with radius over $[0,0.5 R]$ and angle over $[-\pi / 2, \pi / 2]$. As an example, NLOS errors are generated according to the CDSM. We run the proposed method 50 times independently. Figure 10 shows the true scale factors and estimated scale factors for the two BSs. From this figure, we know that the estimated scale factors by using the proposed location method are consistent with the true scale factor to some degree. The estimated scale factor for BS2 is closer to the true scale factor, as shown in Figure 10(b). It is reasonable to use the estimated scale factors to evaluate the level of NLOS propagation.

The AOA of an MS in this paper is estimated from the uplink signal at BSs. So, the proposed method is network based. Once the MS position is determined, AOA seen at BSs may be further corrected. The corrected AOA can be used in downlink beamforming to help the antenna array to distribute power to the MS more accurately. The corrected AOA is also useful to track MSs while they are moving in the cellu-

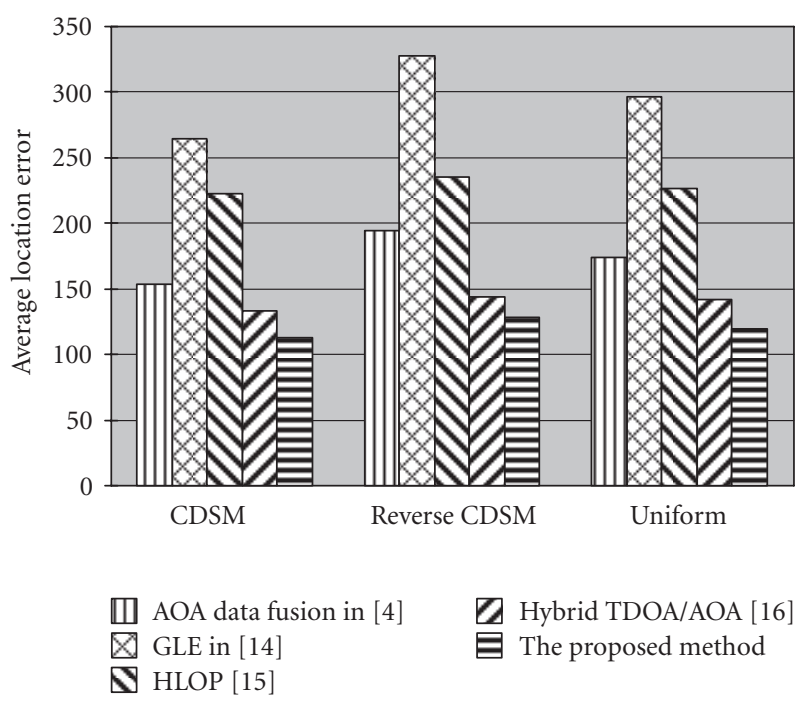

FIGURE 9: The average location errors with scenario 1 on the CDSM, the reverse CDSM, and the uniform NLOS models.

TABLE 1: The standard deviation of the corrected AOA error (in degrees).

\begin{tabular}{cccc}
\hline & CDSM & Reverse CDSM & Uniform \\
\hline$\Delta \theta_{1}$ & 5.49 & 6.09 & 5.99 \\
$\Delta \theta_{2}$ & 4.33 & 5.25 & 4.87 \\
\hline
\end{tabular}

lar network. In this simulation, the MS position is assumed to be located at $(500,866)$ in meter, angle spread is modeled as a Gaussian distribution and the corresponding standard deviation is about 6 degrees, determined by the CDSM. Let $\Delta \theta_{1}$ and $\Delta \theta_{2}$ be the standard deviations of corrected AOA error. Each standard deviation is calculated from 1000 independent runs. Table 1 shows the performance of AOA correction. It is found that $\Delta \theta_{1}$ is almost equal to 6 degrees, but $\Delta \theta_{2}$ is smaller than 6 degrees. That is, the corrected AOA seen at BS1 does not experience any improvement nor degradation, but the corrected AOA seen at BS2 improves.

To demonstrate the performance dependence on the MS position of the proposed method, the ALE is studied by varying the MS locations for the cell layout as shown in Figure 3. The results are illustrated in Figure 11, where the horizontal axis and the vertical axis denote the LOS AOA and LOS TOA ranges, respectively. Figure 11 is the results of the CDSM. The ALE in Figure 11(a) is drawn in 3D space and Figure 11(b) is the corresponding contour. Each ALE in the figure is calculated from 1000 independent runs. Both the two plots prove that the performance of the proposed method is dependent on the MS position. The ALE is not in the same level while the MS position varies. It is observed that (1) ALE tends to be small while the MS is relatively close to the home station; (2) ALE tends to become small while the MS position is on a circle of a certain radius, for example, ALE is small in this simulation while the MS is on a circle with a radius of about 350 meters; (3) ALE tends to become large while the MS is far away from the home BS and far away from 0 degree; (4) 


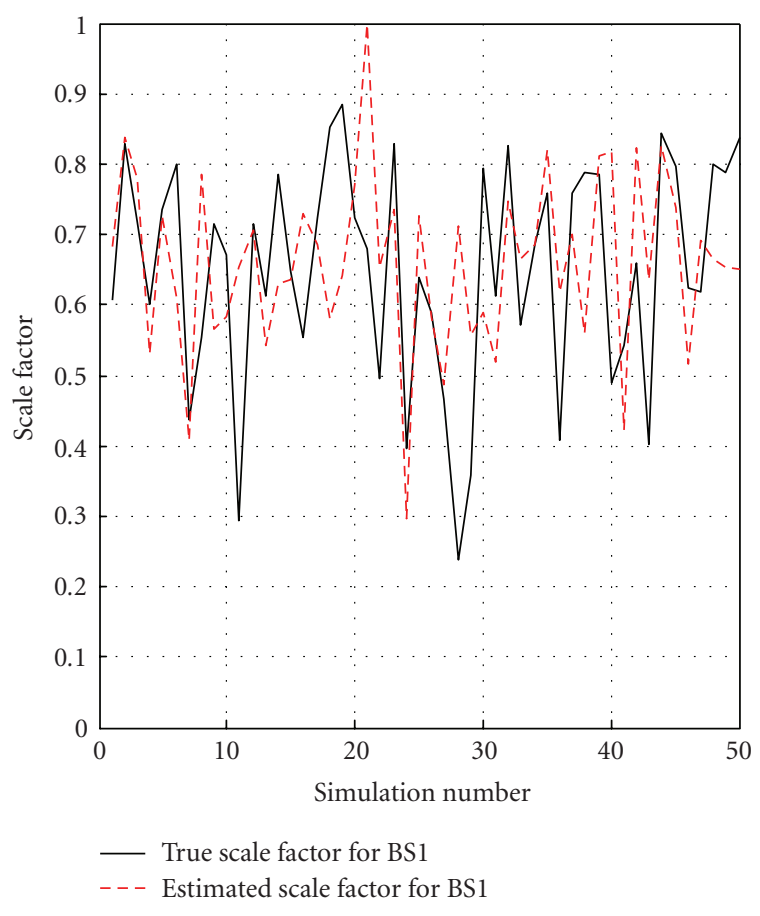

(a)

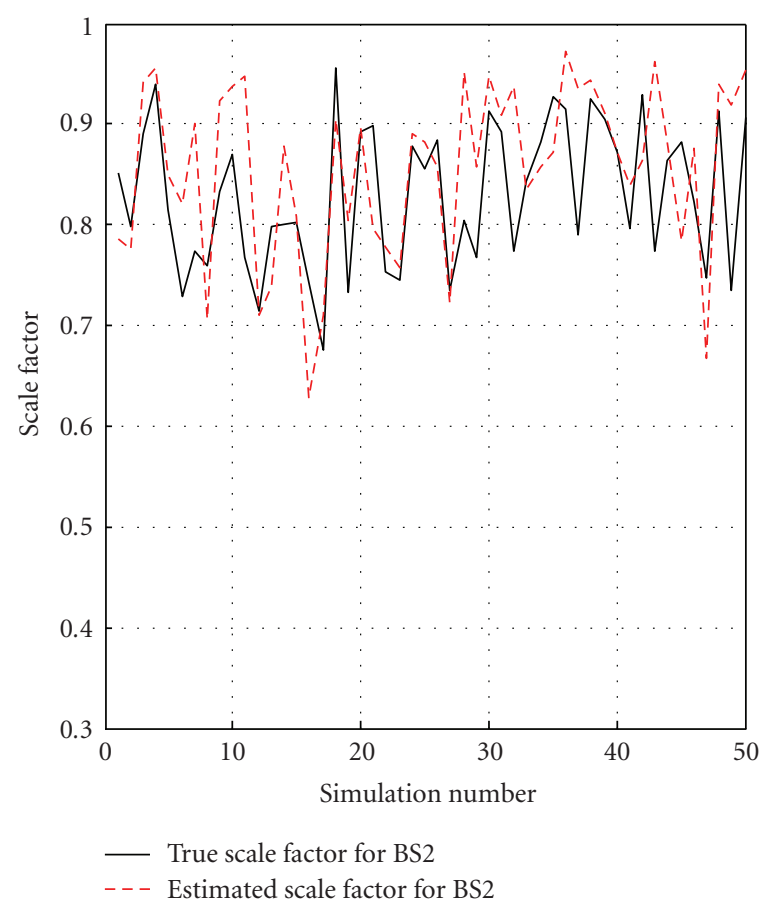

(b)

FIGURE 10: The true scale factor and the estimated scale factor on the CDSM.

a global minimum and two local minima exist. The position $\mathrm{A}$ is the global minimum; positions $\mathrm{B}$ and $\mathrm{C}$ are the two local minima. If look-up tables can be constructed based on these special properties, it is possible to predict location estimation accuracy when the MS location is roughly known. Simulations show that the ALE contour is dependent on the NLOS error model. The positions of these minima may vary depending on the NLOS model.

\subsection{Scenario 2: 3 BSs}

The cell layout of the three BSs scenario is shown in Figure 12. The coordinates of BS1, BS2, and BS2 are $(0,0)$, $(R, 0)$, and $(R / 2, \sqrt{3} R / 2)$, respectively, where $R=2000$ meters. The MS position is assumed to be uniformly distributed in the region formed by the points $\mathrm{O}_{1}, \mathrm{I}, \mathrm{J}$, and $\mathrm{K}$. This assumption is reasonable because the range measurements are considered only from the three nearest neighbor BSs. If the MS is outside this region, another neighbor BS, not considered here, will be closer. Computer simulations are performed to access the performance of these methods, including the GLE in [15], the HLOP in [16], the hybrid TDOA/AOA method in [17], AOA data fusion in [4], TOA data fusion in [4], the method in [9], and the proposed method. For the hybrid TDOA/AOA method, range difference is directly calculated by TOA range measurements, that is, $r_{i 1}=r_{i}-r_{1}$. Figure 13 shows the ALEs of these methods. The TOA data fusion in [4] is a well-known TOA location method and the method in [9] is a modified TOA location method by quadratic programming technique. Both of the two purely TOA methods are used as benchmarks to show performance improvement with angle. Again, we find that ALEs of all the methods are larger on reverse CDSM. The AOA data fusion has excellent performance and is easy to implement. It is found that the proposed method outperform the others.

\section{REMARKS AND CONCLUSIONS}

Motivated by the temporal-spatial characteristics of the radio propagation channel, an effective NLOS error mitigation method is proposed in this paper. The MS position is constrained to a patch overlapped by TOA range measurements and angular bounds. The closed-form equation of the MS position, TOAs, AOAs, and angle spreads is built. The solution space of the closed-form equation becomes large because the angle spreads are random variables in nature. A constrained objective function is constructed to further limit the MS position. A Lagrange-based solution and a numerical solution are proposed to resolve the MS position. It is observed that the proposed method is characterized by the following features.

(i) It is applicable in the case when only two BSs are involved in the location process. Although there are some other methods [4, 15-19] available, they are not specially designed for this scenario and seldom consider the temporal-spatial characteristics of the propagation channel. The proposed method fully considers the temporal-spatial characteristics of the propagation channel and has better performance. Furthermore, it can be easily extended to more than BSs scenario, as illustrated in Section 5. 


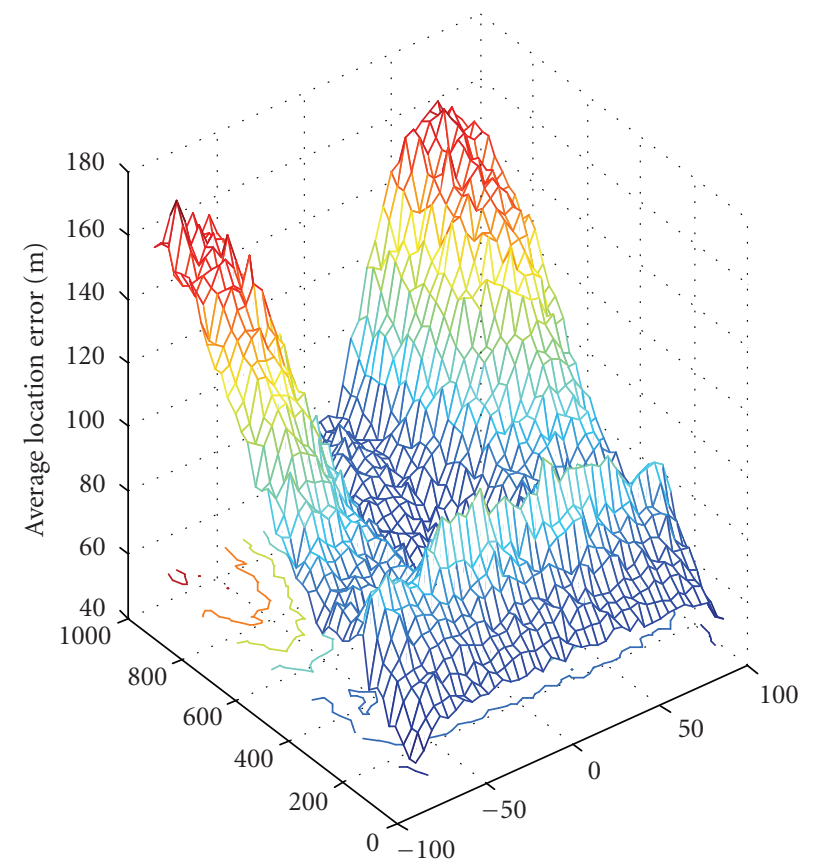

(a)

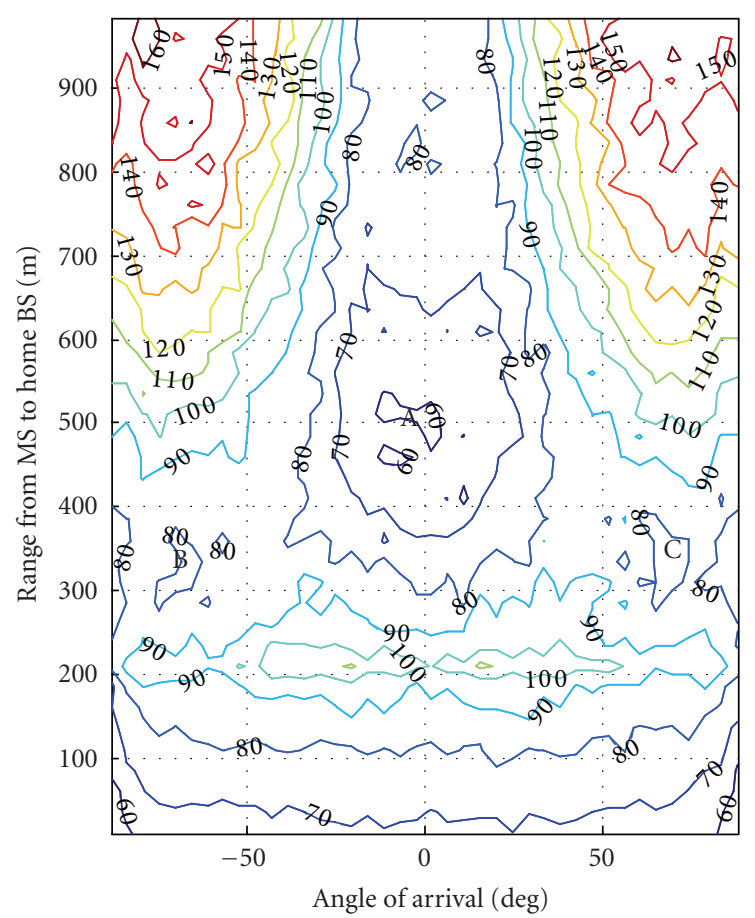

(b)

FIgURE 11: The performance dependence on the MS position on the CDSM. The horizontal axis of (a) is the LOS AOA seen at BS1 and the vertical axis is the LOS distance to BS1.

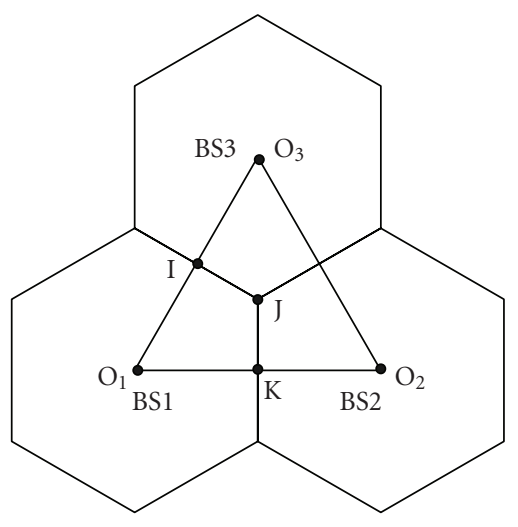

FIGURE 12: The cell layout for scenario 2.

(ii) The angular bounds $\theta_{\min , i}$ and $\theta_{\max , i}$ are the only required prior knowledge. They can be determined by the statistics of AOA distribution from a theoretical model or determined by experimental test data in a real environment. Even the sector information may be considered as a substitute for the angular bounds. However, the methods in [15-19] need more prior knowledge, such as the covariance of TOA (TDOA) measurements, the standard deviation of AOA measurements, and the variance of measurement noise. In a macrocell environment as shown in Figure 1, the angular bounds can be definitely determined by (3).

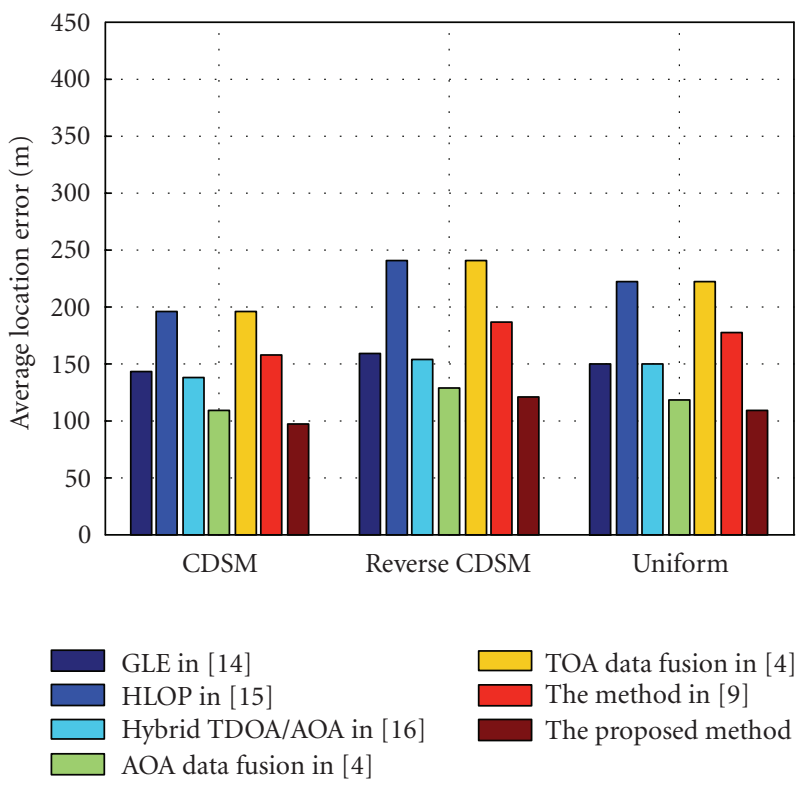

FIgure 13: The average location errors with scenario 2 on the CDSM, reverse CDSM, and uniform models.

But (3) may be not efficient in a microcell environment where AOA seen at the BS has a large spread over $[0,2 \pi]$. So, the proposed method is more applicable in an open outdoor environment. 
(iii) TOA and AOA measurements in this paper are obtained at BSs. So the proposed method is network based. This means that no modification is needed on MSs but software update is required at BSs.

(iv) The smart antenna array is standard equipment at each BS in the TD-SCDMA network [34]. AOA of each MS can be estimated by the uplink beamforming technique. So, the proposed method is attractive for MS location in the TD-SCDMA network. It is also possible to realize the location method in other cellular systems if the azimuth can be obtained.

The performance improvements of the proposed method compared to other hybrid methods are examined by computer simulations. The scale factor can be estimated by the proposed method, which is useful to evaluate NLOS level. The corrected AOA seen at BS1 does not experience any improvement nor degradation. But the corrected AOA seen at BS2 improves. The location accuracy of the proposed method is not only dependent on the MS position but also dependent on NLOS error models.

Finally, we must mention that the proposed method is mainly based on the joint geometric constraints of excess delay and angle spread and does not make full use of the pdf information about them. So, the method is suboptimal-not an optimal statistical one.

\section{ACKNOWLEDGMENTS}

The authors are very grateful to the anonymous reviewers for their useful comments. This work was supported, in part, by the coresearch project of KOSEF/NSFC (Korea Science and Engineering Foundation, the National Natural Science Foundation of China), Korea University ITRC (Information Technology Research Center) project, and the Daegu Gyeongbuk Institute of Science and Technology (DGIST).

\section{REFERENCES}

[1] J. Caffery Jr. and G. L. Stüber, "Overview of radiolocation in CDMA cellular systems," IEEE Communications Magazine, vol. 36, no. 4, pp. 38-45, 1998.

[2] K. Axel., Location-Based Services: Fundamentals and Operation, John Wiley \& Sons, Hoboken, NJ, USA, 2005.

[3] F. Gustafsson and F. Gunnarsson, "Mobile positioning using wireless networks: possibilities and fundamental limitations based on available wireless network measurements," IEEE Signal Processing Magazine, vol. 22, no. 4, pp. 41-53, 2005.

[4] A. H. Sayed, A. Tarighat, and N. Khajehnouri, "Network-based wireless location: challenges faced in developing techniques for accurate wireless location information," IEEE Signal Processing Magazine, vol. 22, no. 4, pp. 24-40, 2005.

[5] K. W. Cheung, H. C. So, W.-K. Ma, and Y. T. Chan, "A constrained least squares approach to mobile positioning: algorithms and optimality," EURASIP Journal on Applied Signal Processing, vol. 2006, Article ID 20858, 23 pages, 2006.

[6] M. I. Silventoinen and Rantalainen, "Mobile station emergency locating in GSM," in Proceedings of the IEEE International Conference on Personal Wireless Communications (ICPWC'96), pp. 232-238, New Delhi, India, February 1996.
[7] M. P. Wylie and J. Holtzman, "Non-line of sight problem in mobile location estimation," in Proceedings of the Annual International Conference on Universal Personal CommunicationsRecord, vol. 2, pp. 827-831, Cambridge, Mass, USA, September 1996.

[8] X. Wang, Z. Wang, and B. O'Dea., "A TOA-based location algorithm reducing the errors due to the non-line-of-sight (NLOS) propagation," IEEE Transactions on Vehicular Technology, vol. 52, pp. 112-116, 2003.

[9] W. Wei, X. Jin-Yu, and Z. Zhong-Liang, "A new NLOS error mitigation algorithm in location estimation," IEEE Transactions on Vehicular Technology, vol. 54, no. 6, pp. 2048-2053, 2005.

[10] S. Venkatraman, J. Caffery Jr., and H.-R. You, "A novel ToA location algorithm using LoS range estimation for NLoS environments," IEEE Transactions on Vehicular Technology, vol. 53, no. 5, pp. 1515-1524, 2004.

[11] Y.-T. Chan, W.-Y. Tsui, H.-C. So, and P.-C. Ching, "Timeof-arrival based localization under NLOS conditions," IEEE Transactions on Vehicular Technology, vol. 55, no. 1, pp. 17-24, 2006.

[12] J. Caffery Jr., "New approach to the geometry of TOA location," in Proceedings of the 52nd IEEE Vehicular Technology Conference (VTC '02), vol. 4, pp. 1943-1949, Boston, Mass, USA, September 2000.

[13] S. Al-Jazzar, J. Caffery Jr., and H.-R. You, "Scattering-modelbased methods for TOA location in NLOS environments," IEEE Transactions on Vehicular Technology, vol. 56, no. 2, pp. 583-593, 2007.

[14] M. Nájar, J. M. Huerta, J. Vidal, and J. A. Castro, "Mobile location with bias tracking in non-line-of-sight," in Proceedings of the IEEE International Conference on Acoustics, Speech and Signal Processing (ICASSP '04), vol. 3, pp. 956-959, 2004.

[15] C.-L. Chen and K.-T. Feng, "An efficient geometryconstrained location estimation algorithm for NLOS environments," in Proceedings of the International Conference on Wireless Networks, Communications and Mobile Computing (IWCMC '07), vol. 1, pp. 244-249, Las Vegas, Nev, USA, June 2005.

[16] S. Venkatraman and J. Caffery Jr., "Hybrid TOA/AOA techniques for mobile location in non-line-of-sight environments," in Proceedings of the IEEE Wireless Communications and Networking Conference (WCNC '04), vol. 1, pp. 274-278, March 2004.

[17] C. Li and Z. Weihua, "Hybrid TDOA/AOA mobile user location for wideband CDMA cellular systems," IEEE Transactions on Wireless Communications, vol. 1, no. 3, pp. 439-447, 2002.

[18] E. Grosicki, K. Abed-Meraim, and R. Dehak, "A novel method to fight the non-line-of-sight error in AOA measurements for mobile location," in Proceedings of the IEEE International Conference on Communications (ICC '04), vol. 5, pp. 2794-2798, June 2004.

[19] M. S. Cugno and F. Barcelo-Arroyo, "WLAN indoor positioning based on TOA with two reference points," in Proceedings of the IEEE 4th Workshop on Position, Navigation and Communication, pp. 23-28, Hannover, Germany, March 2007.

[20] N. Iwakiri and T. Kobayashi, "Joint TOA and AOA estimation of UWB signal using time domain smoothing," in Proceedings of the 2nd International Symposium on Wireless Pervasive Computing, pp. 120-125, San Juan, Puerto, February 2007.

[21] H. Tang and Y. Park, "Location tracking of mobile stations in TD-SCDMA system," in Proceedings of the 4th IEEE Workshop on Positioning, Navigation, and Communication, pp. 153-159, Hannover, Germany, March 2007. 
[22] A. J. Weiss, "Direct position determination of narrowband radio frequency transmitters," IEEE Signal Processing Letters, vol. 11, no. 5, pp. 513-516, 2004.

[23] R. B. Ertel and J. H. Reed, "Angle and time of arrival statistics for circular and elliptical scattering models," IEEE Journal on Selected Areas in Communications, vol. 17, no. 11, pp. 18291840, 1999.

[24] K. I. Pedersen, P. E. Mogensen, and B. H. Fleury, "A stochastic model of the temporal and azimuthal dispersion seen at the base station in outdoor propagation environments," IEEE Transactions on Vehicular Technology, vol. 49, no. 2, pp. 437$447,2000$.

[25] 3GPP/3GPP2 joint spatial channel modeling Ad-hoc (SCM128-Motorola), "Circular angle spread," in Teleconference, March 2003.

[26] M. J. Gans, "Power-spectral theory of propagation in the mobile-radio environment," IEEE Transactions on Vehicular Technology, vol. 21, no. 1, pp. 27-38, 1972.

[27] Y. Jeong, H. You, and C. Lee, "Calibration of NLOS error for positioning systems," in Proceedings of the IEEE Vehicular Technology Conference (VTC '01), vol. 4, no. 53, pp. 2605-2608, May 2001.

[28] L. J. Greenstein, V. Erceg, Y. S. Yeh, and M. V. Clark, "A new path-gain/delay-spread propagation model for digital cellular channels," IEEE Transactions on Vehicular Technology, vol. 46, no. 2, pp. 477-485, 1997.

[29] J. J. Caffery Jr. and G. L. Stüber, "Subscriber location in CDMA cellular networks," IEEE Transactions on Vehicular Technology, vol. 47, no. 2, pp. 406-416, 1998.

[30] I. Jami and R. F. Ormondroyd, "Joint angle of arrival and angle-spread estimation of multiple users using an antenna array and a modified MUSIC algorithm," in Proceedings of the IEEE Vehicular Technology Conference (VTC '01), vol. 1, pp. 48-52, May 2001.

[31] S. Sakagami, S. Aoyama, K. Kuboi, S. Shirota, and A. Akeyama, "Vehicle position estimates by multibeam antennas in multipath environments," IEEE Transactions on Vehicular Technology, vol. 41, no. 1, pp. 63-68, 1992.

[32] "Service provided by the physical layer," Version 3.0.0, China Wireless Telecommunication Standard (CWTS) Working Group 1 (WG1).

[33] "Physical Layer-Measurements," Version 2.0.0, China Wireless Telecommunication Standard (CWTS) Working Group 1 (WG1).

[34] "Physical layer Procedures," Version 3.0.0, China Wireless Telecommunication Standard (CWTS) Working Group 1 (WG1). 